\title{
Pseudocontinuations and the Backward Shift
}

\section{Alexandru Aleman, Stefan Richter $\mathcal{6}$ William T. RosS}

ABstract. In this paper, we will examine the backward shift operator $L f=(f-f(0)) / z$ on certain Banach spaces of analytic functions on the open unit disk $\mathbb{D}$. In particular, for a (closed) subspace $\mathcal{M}$ for which $L \mathcal{N} \subset \mathcal{N}$, we wish to determine the spectrum, the point spectrum, and the approximate point spectrum of $L \mid \mathcal{M}$. In order to do this, we will use the concept of "pseudocontinuation" of functions across the unit circle $\mathbb{T}$.

We will first discuss the backward shift on a general Banach space of analytic functions and then for the weighted Hardy and Bergman spaces, we will show that $\sigma(L \mid \mathcal{M})=\sigma_{\text {ap }}(L \mid \mathcal{M})$ and moreover whenever $\mathcal{M}$ does not contain all of the polynomials, then

$$
\sigma(L \mid \mathcal{M}) \cap \mathbb{D}=\sigma_{p}(L \mid \mathcal{M}) \cap \mathbb{D}=\sigma_{\text {ap }}(L \mid \mathcal{M}) \cap \mathbb{D}
$$

and is a Blaschke sequence. In fact, for certain measures, we will show that $\mathcal{M}$ is contained in the Nevanlinna class and every function in $\mathcal{M}$ has a pseudocontinuation across $\mathbb{T}$ to a function in the Nevanlinna class of the exterior disk.

For the Dirichlet and Besov spaces however, the spectral picture of $\sigma(L \mid \mathcal{M})$ is quite different. For example $\sigma_{\mathrm{ap}}(L \mid \mathcal{M})$ and $\sigma(L \mid \mathcal{M})$ can differ and even when

$$
\sigma(L \mid \mathcal{M}) \cap \mathbb{D}=\sigma_{p}(L \mid \mathcal{M}) \cap \mathbb{D}=\sigma_{\text {ap }}(L \mid \mathcal{M}) \cap \mathbb{D}
$$

and is discrete, it need not be a Blaschke sequence. Moreover, $\mathcal{M}$ may contain functions which do not have pseudocontinuations across any set of positive measure in $\mathbb{T}$.

As an application of our pseudocontinuation techniques and the so-called " $H^{2}$-duality", we will look at the index of the $M_{z}$-invariant subspaces of the Bergman spaces and weighted Dirichlet spaces. In particular, whenever $f$ and $g$ belong to 
the unweighted Bergman space $L_{a}^{p}(\mathbb{D})$ and $f / g$ has finite nontangential limits almost everywhere on a set of positive Lebesgue measure in the circle, then the $M_{z}$-invariant subspace generated by $f$ and $g$ has index equal to one. For a large class of weighted Dirichlet spaces, we will show that every non-zero $M_{z}$-invariant subspace has index equal to one.

\section{IntRoduCtion AND STATEMENT OF THE MAIN RESUlts}

Let $\mathcal{B}$ be a Banach space of analytic functions defined on the open unit disk $\mathbb{D}=\{|z|<1\}$ for which the backward shift operator

$$
L f=\frac{f-f(0)}{z}, f \in \mathcal{B}
$$

is continuous. Backward shift operators and their restrictions $L \mid \mathcal{M}$ to invariant subspaces $\mathcal{M} \subset \mathcal{B}^{1}$ form a large class of examples of bounded linear operators. The main body of the book [33] contains an extensive study of these operators in the case when $\mathcal{B}$ equals the Hardy space $H^{2}$. For other spaces $\mathcal{B}$, various authors have investigated different aspects of the invariant subspace structure of the backward shift [1] [2] [3] [4] [8] [20] [38].

In this paper, for certain Banach spaces $\mathcal{B}$, we will relate meromorphic continuations of functions in the $L$-invariant subspaces $\mathcal{M}$ of $\mathcal{B}$ to the spectrum of $L \mid \mathcal{M}$. In fact, an elementary computation shows that if $\lambda \in \mathbb{C}$ is such that $(I-\lambda L) \mid \mathcal{M}$ is invertible, then for all $f \in \mathcal{M}$

$$
(I-\lambda L)^{-1} f=\frac{z f-\lambda c_{\lambda}(f)}{z-\lambda}
$$

for some constant $c_{\lambda}(f) \in \mathbb{C}$. If $\lambda \in \mathbb{D}$, then by analyticity $c_{\lambda}(f)=f(\lambda)$. It turns out that for many choices of spaces $\mathcal{B}$ and proper $L$-invariant subspaces $\mathcal{M}$, the functions $f \in \mathcal{M}$ have meromorphic 'continuations' $\tilde{f}$ in the exterior disk, and $c_{\lambda}(f)=\tilde{f}(\lambda)$ if $(I-\lambda L) \mid \mathcal{M}$ is invertible. If $\sigma(L \mid \mathcal{M})$ omits an arc $I$ in the unit circle $\mathbb{T}$, then $\tilde{f}$ is an ordinary analytic continuation of $f$ across $I$ and this is well known. However, our results will cover many cases where $\sigma(L \mid \mathcal{M})$ may contain the whole unit circle. In some of those cases, we shall see that the meromorphic continuations are 'pseudocontinuations' in the sense of H. S. Shapiro [42]. Sometimes we will have to employ a continuation concept that is even weaker then that of a pseudocontinuation. To make this all precise, we proceed as follows:

Let $\mathcal{B}$ be a Banach space of analytic functions on $\mathbb{D}$ which satisfies the following five properties:

$$
\mathcal{B} \hookrightarrow \operatorname{Hol}(\mathbb{D})
$$

1 Throughout this paper, a subspace will always be a closed linear manifold. 


$$
\begin{gathered}
M_{z} \mathcal{B} \subset \mathcal{B}, M_{z} f=z f \\
1 \in \mathcal{B} \\
L_{\lambda} \mathcal{B} \subset \mathcal{B}, \quad \forall \lambda \in \mathbb{D}, L_{\lambda} f=\frac{f-f(\lambda)}{z-\lambda} \\
\sigma\left(M_{z}\right)=\mathbb{D}^{-} .
\end{gathered}
$$

Remark 1.1. In (1.1), the inclusion map from $\mathcal{B}$ (with the norm topology) to $\operatorname{Hol}(\mathbb{D})$ (with the topology of uniform convergence on compact sets) is both injective and continuous.

Examples include the Hardy spaces, the weighted Bergman spaces, the weighted Dirichlet spaces, and the Besov spaces (see below for the definitions of these spaces). It follows from (1.1), (1.2), (1.4), and the closed graph theorem that the operators $M_{z}$ and $L_{\lambda}$ are continuous on $\mathcal{B}$. We will denote the collection of $L$-invariant subspaces by $\operatorname{Lat}(L, \mathcal{B})$.

In this general setting, one can prove (see Section 2) that

$$
\begin{gathered}
\sigma(L)=\mathbb{D}^{-}, \\
\sigma(L \mid \mathcal{M}) \subset \mathbb{D}^{-} \quad \forall \mathcal{M} \in \operatorname{Lat}(L, \mathcal{B}), \\
\sigma_{\mathrm{ap}}(L \mid \mathcal{M}) \cap \mathbb{D}=\sigma_{p}(L \mid \mathcal{M}) \cap \mathbb{D}=\left\{a \in \mathbb{D}: \frac{1}{1-a z} \in \mathcal{M}\right\} .
\end{gathered}
$$

Moreover, the set in (1.6) is either discrete or all of $\mathbb{D}$. In fact this set is all of $\mathbb{D}$ if and only if $\mathcal{M}$ contains all of the polynomials. Under a mild regularity condition on $\mathcal{B}$, one can even prove that

$$
\sigma_{\mathrm{ap}}(L \mid \mathcal{M}) \cap \mathbb{T}=\sigma(L \mid \mathcal{M}) \cap \mathbb{T}
$$

and is the complement (in the unit circle) of the set of points $\zeta \in \mathbb{T}$ such that every $f \in \mathcal{M}$ extends to be analytic in a neighborhood of $1 / \zeta$. Furthermore since $\partial \sigma(L \mid \mathcal{M}) \subset \sigma_{\text {ap }}(L \mid \mathcal{M})$, then one can argue that

either $\sigma(L \mid \mathcal{M}) \cap \mathbb{D}=\sigma_{\text {ap }}(L \mid \mathcal{M}) \cap \mathbb{D}$ and is discrete

$$
\text { or } \sigma(L \mid \mathcal{M})=\mathbb{D}^{-} .
$$

For the weighted Hardy and Bergman spaces, it will turn out that $\sigma(L \mid \mathcal{M})=$ $\sigma_{\text {ap }}(L \mid \mathcal{M})$ and when $\sigma(L \mid \mathcal{M}) \cap \mathbb{D}$ is discrete, it is a Blaschke sequence. For other spaces, such as the Besov spaces and weighted Dirichlet spaces, $\sigma(L \mid \mathcal{M})$ can 
differ from $\sigma_{\text {ap }}(L \mid \mathcal{M})$. Moreover, even when $\sigma(L \mid \mathcal{M}) \cap \mathbb{D}=\sigma_{\text {ap }}(L \mid \mathcal{M}) \cap \mathbb{D}$ and is discrete, it need not be a Blaschke sequence.

The main tool that will be used to determine $\sigma(L \mid \mathcal{M})$ is this next simple observation for which we will adhere to the following convention: If $\mathcal{B}^{*}$ denotes the dual of $\mathcal{B}$, then for $\phi \in \mathcal{B}^{*}$ we will write the action of a linear functional on $\mathcal{B}$ by $f \rightarrow\langle f, \phi\rangle$.

Proposition 1.2. If $|\lambda|>1$ with $1 / \lambda \notin \sigma_{\mathrm{ap}}(L \mid \mathcal{M})$ then $((I-\lambda L) \mid \mathcal{M})^{-1}$ exists if and only if for every $f \in \mathcal{M}$, the quantity

$$
c_{\lambda}(f, \phi)=\left\langle\frac{z f}{z-\lambda}, \phi\right\rangle /\left\langle\frac{\lambda}{z-\lambda}, \phi\right\rangle
$$

is independent of the choice of $\phi \in \mathcal{M}^{\perp}$ with $\left\langle(z-\lambda)^{-1}, \phi\right\rangle \neq 0$. In fact, if $|\lambda|>1$ with $1 / \lambda \notin \sigma(L \mid \mathcal{M})$, then $c_{\lambda}(f, \phi)=c_{\lambda}(f)$ and

$$
((I-\lambda L) \mid \mathcal{M})^{-1} f=\frac{z f-\lambda c_{\lambda}(f)}{z-\lambda}
$$

for all $f \in \mathcal{M}$ and $\phi \in \mathcal{M}^{\perp}$ with $\left\langle(z-\lambda)^{-1}, \phi\right\rangle \neq 0$.

Note that from (1.6) such $\phi$ 's exist. Also note that whenever $|\lambda|>1$, $1 / \lambda \notin \sigma_{\mathrm{ap}}(L \mid \mathcal{M})$, and $\phi \in \mathcal{M}^{\perp}$ with $\left\langle(z-\lambda)^{-1}, \phi\right\rangle \neq 0$, then the function

$$
\xi \rightarrow c_{\xi}(f, \phi)
$$

is meromorphic on the exterior disk (with possible poles at the points $\xi$, where $\xi^{-1} \in \sigma_{\mathrm{ap}}(L \mid \mathcal{M}) \cap \mathbb{D}$. Note that by (1.6) this set is discrete). One way to show that $c_{\lambda}(f, \phi)$ is independent of $\phi$ is to show that the finite non-tangential limits of the function $\xi \rightarrow c_{\xi}(f, \phi)$ (on the exterior disk) are equal to the finite nontangential limits of $f$ (on the disk) on some set of positive Lebesgue measure on the circle. ${ }^{2}$ Then using Privalov's uniqueness theorem for meromorphic functions [32], p. $84-86, c_{\lambda}(f, \phi)$ would be independent of the choice of $\phi$. In this case, the two functions would be pseudocontinuations of each other, a concept we will define below. We illustrate this idea with the following two examples.

Example 1.3. For $1<p<+\infty$, let $\mathcal{B}=H^{p}$ denote the Hardy space of analytic functions $f$ on $\mathbb{D}$ for which

$$
\sup _{0<r<1} \int_{|\zeta|=1}|f(r \zeta)|^{p} \frac{|d \zeta|}{2 \pi}<+\infty
$$

2 Of course, one needs to show that for the particular Banach space in question, these two non-tangential limits indeed exist since there is no a priori reason why they should. 
Conditions (1.1) through (1.5) are well known for $H^{p}$ [21]. Furthermore, it is known [21], Theorem 7.3, that the dual of $H^{p}$ can be identified with $H^{q}$ (where $q$ is the conjugate index to $p$ ) via the pairing

$$
\langle f, g\rangle=\lim _{r \rightarrow 1^{-}} \int_{|\zeta|=1} f(r \zeta) \overline{g(r \zeta)} \frac{|d \zeta|}{2 \pi} .
$$

Moreover, it can be shown that for $\mathcal{M} \in \operatorname{Lat}\left(L, H^{p}\right), \mathcal{M} \neq H^{p}$, and $|\lambda|>1$, the constant $c_{\lambda}(f, g)$ does not depend on $g \in H^{q}$ with

$$
g \in \mathcal{M}^{\perp} \text { and }\left\langle\frac{1}{z-\lambda}, g\right\rangle \neq 0 .
$$

To prove this result, one uses [21], p. 39, to show that for any $g \in H^{q}$ satisfying (1.7), the meromorphic function

$$
\xi \rightarrow\left\langle\frac{z f}{z-\xi}, g\right\rangle /\left\langle\frac{\xi}{z-\xi}, g\right\rangle, \quad \xi \in\{|z|>1\} \cup\{\infty\}
$$

is in the Nevanlinna class and hence, by Fatou's theorem, has finite non-tangential limits $[|d \zeta|]$-a.e. on $\mathbb{T}$. Moreover using [21], p. 39 (the "jump theorem"), and the fact that $f \in \mathcal{M}$ and $g \in \mathcal{M}^{\perp}$, one proves that these limits are equal $[|d \zeta|]$-a.e. to the non-tangential limits of $f$ on $\mathbb{T}$. By Privalov's uniqueness theorem, $c_{\lambda}(f, g)$ is independent of $g$. We remark that a similar result holds for both $H^{1}$ and the disk algebra.

Example 1.4. Let $\mathcal{B}=L_{a}^{2}$ denote the Bergman space of analytic functions $f$ on $\mathbb{D}$ for which

$$
\int_{\mathbb{D}}|f|^{2} \frac{d A}{\pi}<+\infty
$$

where $d A$ is two-dimensional area measure. Again, one can verify properties (1.1) through (1.5) [16], Chapter 2, Section 8. The dual of $L_{a}^{2}$ can be identified with the Dirichlet space of analytic functions $g$ on $\mathbb{D}$ with finite Dirichlet integral

$$
\int_{\mathbb{D}}\left|g^{\prime}\right|^{2} \frac{d A}{\pi}
$$

via the pairing

$$
\langle f, g\rangle=\lim _{r \rightarrow 1^{-}} \int_{|\zeta|=1} f(r \zeta) \overline{g(r \zeta)} \frac{|d \zeta|}{2 \pi}
$$

(see [31]). For $\mathcal{M} \in \operatorname{Lat}\left(L, L_{a}^{2}\right), \mathcal{M} \neq L_{a}^{2}, f \in \mathcal{M}$, and $g$ in the Dirichlet space satisfying (1.7), one shows (see [38] Theorem 2.2 and Section 6) that the meromorphic function defined by (1.8) is in the Nevanlinna class of the exterior disk. However, unlike for the Hardy space, Bergman functions are not in the Nevanlinna class of $\mathbb{D}$ and hence we are not guaranteed the existence of non-tangential 
(or even radial [29]) limits for $f$. However, S. Richter and C. Sundberg [38] overcome this difficulty and show that in fact $\mathcal{M}$ is contained in the Nevanlinna class of $\mathbb{D}$ and moreover the non-tangential limits of $f$ are equal to the non-tangential limits of the function (1.8) $[|d \zeta|]$-a.e. Again using Privalov's uniqueness theorem, $c_{\lambda}(f, g)$ is independent of $g$.

Thus we see in these two cases that whenever $\mathcal{M} \in \operatorname{Lat}(L, \mathcal{B}), \mathcal{M} \neq \mathcal{B}$, and $f \in \mathcal{M}$, then $c_{\lambda}(f)=f(\lambda)$ when $|\lambda|<1$ and the function $\lambda \rightarrow c_{\lambda}(f)$ is a Nevanlinna function on $\{|z|>1\} \cup\{\infty\}$ whose non-tangential limits equal those of $f[|d \zeta|]$-a.e. on $\mathbb{T}$. Furthermore, in these two cases

$$
\sigma(L \mid \mathcal{M}) \cap \mathbb{D}=\sigma_{\text {ap }}(L \mid \mathcal{M}) \cap \mathbb{D}=\sigma_{p}(L \mid \mathcal{M}) \cap \mathbb{D}=\left\{a \in \mathbb{D}: \frac{1}{1-a z} \in \mathcal{M}\right\}
$$

and moreover, this set must either be discrete or all of $\mathbb{D}$. This type of "extension" of a meromorphic function across $\mathbb{T}$ as above is called a pseudocontinuation (in the sense of H.S. Shapiro [42]) and will be the main technique used to show that $c_{\lambda}(f, \phi)$ is independent of $\phi$. For the statement our main results we make the following definitions.

\section{Definition 1.5.}

(1) Let $\mathbb{D}$ represent the open unit disk and $\mathbb{D}_{e}=\mathbb{C}_{\infty} \backslash \mathbb{D}^{-}$represent the extended exterior disk.

(2) Let $\mathfrak{M}(\mathbb{D})$ and $\mathfrak{M}\left(\mathbb{D}_{e}\right)$ denote the set the meromorphic functions on $\mathbb{D}$ and $\mathbb{D}_{e}$ respectively, and $\mathfrak{N}(\mathbb{D})$ and $\mathfrak{N}\left(\mathbb{D}_{e}\right)$ denote the set of Nevanlinna functions on $\mathbb{D}$ and $\mathbb{D}_{e}$ respectively (i.e., meromorphic functions which can be represented as the quotient of two bounded analytic functions).

(3) For a set $E \subset \mathbb{T}$ with $|E|>0(|E|$ represents the Lebesgue measure of $E$ on the unit circle), we say a function $G \in \mathfrak{M}\left(\mathbb{D}_{e}\right)$ is a pseudocontinuation of $g \in \mathfrak{M}(\mathbb{D})$ across $E$ if the non-tangential limits of $G$ and $g$ exist and are equal $[|d \zeta|]$-a.e. on $E$.

Remark 1.6. By Privalov's uniqueness theorem for meromorphic functions [32], p. $84-86$, whenever a pseudocontinuation (across $E,|E|>0$ ) exists, it is unique.

\section{Example 1.7.}

(1) If $f$ is an inner function, then

$$
\tilde{f}(z)=\frac{1}{\bar{f}(1 / \bar{z})}, \quad \mathbb{D}_{e} \backslash\{z: f(1 / \bar{z})=0\}
$$

is a pseudocontinuation of $f$ across $\mathbb{T}$. We also point out that if the zeros of the inner function $f$ accumulate everywhere on $\mathbb{T}$, then $\tilde{f}$, although a pseudocontinuation, will not be an analytic continuation of $f$. 
(2) The function $e^{z}$ does not have a pseudocontinuation across any set $E \subset$ $\mathbb{T}$ (even though it has an analytic continuation to $\mathbb{C}$ ) since it has an essential singularity at infinity.

We shall now state our results about the spectrum of $L \mid \mathcal{M}$ for the case where the space $\mathcal{B}$ "is" a subspace of $L^{t}(\mu)$ for some $1 \leq t<+\infty$ and $\mu$ a nontrivial finite Borel measure supported in $\mathbb{D}^{-}$. These results will cover both of the examples above as well as some spaces which do not contain the polynomials as a dense subset ${ }^{3}$. Since we want to allow measures which may place mass on $\mathbb{T}$ as well as measures that are carried by a discrete subset of $\mathbb{D}$, the definition of these subspaces is somewhat delicate. We proceed as follows:

Let $\mathcal{B}$ be a Banach space of analytic functions on $\mathbb{D}$ that satisfies properties (1.1) through (1.5). Furthermore, let $1 \leq t<+\infty$ and let $\mu$ be a finite Borel measure whose support is contained in $\mathbb{D}^{-}$and suppose there is a linear isometry

$$
U: \mathcal{B} \rightarrow L^{t}(\mu)
$$

such that

$$
U 1=1 \text { and } U\left(M_{z} \mid \mathcal{B}\right)=\left(M_{z} \mid L^{t}(\mu)\right) U .
$$

Since $\mathcal{B}$ satisfies (1.1), it follows (see Section 4 ) that $\mu \mid \mathbb{T}$ cannot have a singular part, i.e.,

$$
d \mu|\mathbb{T}=g| d \zeta \mid, g \in L^{1}(\mathbb{T},|d \zeta|), g \geq 0 .
$$

Furthermore, we will show (Proposition 4.2) that if $f \in \mathcal{B}$, and

$$
S_{g}=\mathbb{T} \backslash\{\zeta \in \mathbb{T}: g(\zeta)=0\},
$$

then

$$
\begin{gathered}
f|\mathbb{D}=(U f)| \mathbb{D}[\mu] \text {-a.e. } \\
f_{r}\left|S_{g} \rightarrow(U f)\right| S_{g} \text { in measure }[|d \zeta|] \text { as } r \rightarrow 1^{-} .4
\end{gathered}
$$

Thus we may identify $\mathcal{B}$ with the range of $U$ and we shall say that $\mathcal{B}$ is an analytic subspace of $L^{t}(\mu)$.

\section{Remark 1.8 .}

(1) If $\mathcal{B}$ is the Bergman space $L_{a}^{2}$, then $d \mu=d A$ and $U$ is the identity map while if $\mathcal{B}$ is the Hardy space $H^{p}, 1<p<+\infty$, then $d \mu=|d \zeta|$ and $U$ is the isometric map $f|\mathbb{D} \rightarrow f| \mathbb{T}$, where $f \mid \mathbb{T}$ are the non-tangential boundary values of $f \in H^{p}[21]$, p. 21 .

(2) We caution the reader by pointing out that if the measure $\mu$ is carried by a discrete subset of $\mathbb{D}$, then for every $f \in \mathcal{B}$ there will be many distinct analytic functions $g \in \operatorname{Hol}(\mathbb{D})$ such that $f=g[\mu]$-a.e.

\footnotetext{
${ }^{3}$ e.g., By using [30], the polynomials are not dense in $L^{2}\left(\mathbb{D},|\phi|^{2} d A\right) \cap \operatorname{Hol}(\mathbb{D})$, where $\phi$ is an atomic inner function whose singular measure is a point mass at $\zeta=1$.

${ }^{4}$ Here $f_{r}(\zeta)=f(r \zeta)$ for $0 \leq r<1$ and $\zeta \in \mathbb{T}$.
} 
Theorem 1.9. Let $1 \leq t<+\infty$ and let $\mu$ be a non-trivial finite Borel measure on $\mathbb{D}^{-}$such that the space $\mathcal{B}$ is an analytic subspace of $L^{t}(\mu)$. Let $\mathcal{M} \in \operatorname{Lat}(L, \mathcal{B})$ with $\mathcal{M} \neq(0)$. Then

(1) $\sigma(L \mid \mathcal{M})=\sigma_{\mathrm{ap}}(L \mid \mathcal{M})$. More precisely,

(a) $\sigma(L \mid \mathcal{M}) \cap \mathbb{D}=\sigma_{\text {ap }}(L \mid \mathcal{M}) \cap \mathbb{D}=\sigma_{p}(L \mid \mathcal{M}) \cap \mathbb{D}=$ $\left\{a \in \mathbb{D}:(1-a z)^{-1} \in \mathcal{M}\right\}$.

(b) $\sigma(L \mid \mathcal{M}) \cap \mathbb{T}=\sigma_{\text {ap }}(L \mid \mathcal{M}) \cap \mathbb{T}$ and is the following set:

$\mathbb{T} \backslash\{1 / \zeta \in \mathbb{T}:$ every $f \in \mathcal{M}$ extends to be analytic in a neighborhood of $\zeta\}$.

(2) $\sigma(L \mid \mathcal{M})=\mathbb{D}^{-}$if and only if $\mathcal{M}$ contains all of the polynomials.

(3) If $\mathcal{M}$ does not contain all of the polynomials, then $\sigma(L \mid \mathcal{M}) \cap \mathbb{D}$ is a Blaschke sequence. Furthermore, for each $f \in \mathcal{M}$ there exists a unique $\tilde{f} \in \mathfrak{N}\left(\mathbb{D}_{e}\right)$ such that

$$
f_{r}|\mathbb{T} \rightarrow \tilde{f}| \mathbb{T}^{5} \text { in measure }[|d \zeta|] \text { as } r \rightarrow 1^{-} .
$$

Moreover, the function $\tilde{f}$ is given by

$$
\tilde{f}(\lambda)=\int \frac{f \bar{h}}{z-\lambda} d \mu / \int \frac{\bar{h}}{z-\lambda} d \mu,
$$

for all $h \in \mathcal{M}^{\perp}$ which do not annihilate all of the polynomials.

Remark 1.10. It follows from part 3 of this theorem that if a function $f$ in such an $L$-invariant subspace $\mathcal{M}$ has non-tangential limits $[|d \zeta|]$-a.e. on a set of $E \subset \mathbb{T}$ of positive measure, then $\tilde{f}$ is a pseudocontinuation of $f$ across $E$.

For certain measures $\mu$, we can show that such $L$-invariant subspaces $\mathcal{M}$ are in fact contained in the Nevanlinna class of $\mathbb{D}$ and hence by Theorem 1.9 every $f \in \mathcal{M}$ has a pseudo-continuation across all of $\mathbb{T}$. Examples of such measures are the following: Let $\nu$ be a finite measure carried by $[0,1)$, with the additional property that $\nu\{[r, 1)\}>0$ for all $0<r<1$. Let $w \in L^{1+\varepsilon}(d \nu|d \zeta|)$ for some $\varepsilon>0$ be such that $|\psi| \leq w[d \nu|d \zeta|]$-a.e. for some non-zero bounded analytic function $\psi$ on $\mathbb{D}$. Setting

$$
d \mu=w d \nu|d \zeta|
$$

one can show that

$$
\mathcal{B}=L_{a}^{t}(\mu)=L^{t}(\mu) \cap \operatorname{Hol}(\mathbb{D})
$$

satisfies properties (1.1) through (1.5) ${ }^{6}$. Note that such measures are carried by $\mathbb{D}$ and, loosely speaking, are not far away from being radially symmetric. For example, one checks that if $0 \neq \phi \in \mathfrak{N}(\mathbb{D})$ and $\alpha>0$ such that $|\phi|^{\alpha} \in$ $L^{1+\varepsilon}(d \nu|d \zeta|)$, then $d \mu=|\phi|^{\alpha} d \nu|d \zeta|$ satisfies this hypothesis.

5 Here we mean the non-tangential limit values of the Nevanlinna function $\tilde{f}$ on $\mathbb{T}$.

${ }^{6}$ Use a modification of the proof of Lemma 2 in [9]. 
Theorem 1.11. Let $\mu$ be as above and $1 \leq t<+\infty$. If $\mathcal{M} \in \operatorname{Lat}\left(L, L_{a}^{t}(\mu)\right)$ does not contain all of the polynomials, then $\mathcal{M} \subset \mathfrak{N}(\mathbb{D})$ and every $f \in \mathcal{M}$ has a pseudocontinuation across $\mathbb{T}$ to a function in $\mathfrak{N}\left(\mathbb{D}_{e}\right)$.

Remark 1.12. For a function $f \in L_{a}^{t}(\mu)$, we let $[f]_{L}$ denote the smallest $L$-invariant subspace which contains $f$. We say that $f$ is a cyclic vector for $L$ if $[f]_{L}=L_{a}^{t}(\mu)$. The above theorem says that every non-cyclic vector in $L_{a}^{t}(\mu)$ has a pseudocontinuation to a Nevanlinna function on $\mathbb{D}_{e}$. For certain analytic subspaces of $L^{t}(\mu)$ (for example the Hardy spaces $H^{p}$ ) the existence of a Nevanlinna pseudocontinuation is both necessary and sufficient for non-cyclicity. For other spaces such as $L_{a}^{t}\left((1-|z|)^{\alpha} d A\right.$ ), it is not hard to see using duality (see Section 5) that certain inner functions (which always have Nevanlinna pseudocontinuations) are indeed cyclic vectors for $L$.

It is well known (see Section 5) that there is a natural correspondence between the $L$-invariant subspaces of $\mathcal{B}$ and the $M_{z}$-invariant subspaces of a certain "dual" space $\mathfrak{D}$ via the " $H^{2}$-duality" [33]. For example, if $\mathcal{B}$ is the weighted Bergman space $L_{a}^{q}\left((1-|z|)^{\alpha} d A\right)$, then the dual space $\mathfrak{D}$ is the Besov space $X_{\alpha, p}$ (see Section 5 for a definition), and vice versa. We can apply our results about the spectrum of $L \mid \mathcal{M}$ to obtain results about the index of $M_{z}$-invariant subspaces $\mathcal{N}$ of $\mathfrak{D}$. For $\mathcal{N} \in \operatorname{Lat}\left(M_{z}, \mathfrak{D}\right)$ the index of $\mathcal{N}$ is defined by

$$
\operatorname{ind}(\mathcal{N})=\operatorname{dim}(\mathcal{N} / z \mathcal{N}) \text {. }
$$

For example, if $f$ is a non-zero element of $\mathfrak{D}$, then $[f]$, the smallest $M_{z}$-invariant subspace of $\mathfrak{D}$ which contains $f$, has index equal to one. If $\mathfrak{D}$ is the Hardy space $H^{2}$ or the classical Dirichlet space ${ }^{7}$ and $\mathcal{N} \in \operatorname{Lat}\left(M_{z}, \mathfrak{D}\right), \mathcal{N} \neq(0)$, then $\operatorname{ind}(\mathcal{N})=1$ and in fact

$$
\mathcal{N}=\left[\mathcal{N} \cap(z \mathcal{N})^{\perp}\right] .
$$

See [12] [36]. It is known that $\sigma(L \mid \mathcal{M}) \cap \mathbb{D}$ is discrete if and only if the corresponding $M_{z}$-invariant subspace $\mathcal{N}$ has index equal to one ${ }^{8}$. Using our results about the discreteness of the spectrum of $L$-invariant subspaces in Section 4 , we will prove in Section 5 that for a large class of weighted Dirichlet spaces and Besov spaces (which are the "duals" of these spaces) every non-zero $M_{z}$-invariant subspace has index equal to one.

In the next part of the paper, we consider spaces of "smooth" functions, mainly the Besov classes $X_{\alpha, p}$ where the spectral and pseudocontinuation situation is strikingly different (even though these functions are in the Nevanlinna class). This is indeed to be expected since by the " $H^{2}$-duality", these $L$-invariant subspaces correspond to $M_{z}$-invariant subspaces of the weighted Bergman spaces which are known to be very complicated.

\footnotetext{
${ }^{7}$ In both of these examples, $\mathfrak{D}$ is a Hilbert space.

${ }^{8}$ Here we must assume that the polynomials are dense in $\mathcal{B}$.
} 
Remark 1.13. We are grateful to Carl Sundberg, who showed us an argument which yields the following theorem. Our original version was somewhat weaker.

Theorem 1.14. Given $\alpha>-1$ and $1<p<+\infty$, there is a function $f \in X_{\alpha, p}$ such that $[f]_{L} \neq X_{\alpha, p}$ and $f$ has no pseudocontinuation across any set of positive measure in $\mathbb{T}$.

In fact, the same is true for any Banach space of analytic functions $\mathfrak{B}$ which satisfy conditions (1.1) through (1.5) along with the one additional condition that $\mathfrak{B} \hookrightarrow X_{\alpha, p}$ for some $\alpha$ and $p$ (see Section 5 for details). Furthermore (see Proposition 6.1), antipodal to the analytic subspaces of $L^{t}(\mu)$, there are examples of $\mathcal{M} \in \operatorname{Lat}\left(L, X_{\alpha, p}\right)$ for which

$$
\sigma(L \mid \mathcal{M})=\mathbb{D}^{-}, \sigma_{\mathrm{ap}}(L \mid \mathbb{T})=\mathbb{T}, \text { and } \sigma_{p}(L \mid \mathcal{M})=\emptyset .
$$

For $1<p<+\infty$, the $L^{p}$-Dirichlet spaces $\mathcal{D}_{p}$ are defined to consist of all $f \in \operatorname{Hol}(\mathbb{D})$ such that $f^{\prime} \in L^{p}(d A)$. Despite the fact that $\mathcal{D}_{p}=X_{0, p}$ and the above results, we will show in Section 7 that for certain $L$-invariant subspaces $\mathcal{M}$ of $\mathcal{D}_{p}$, there is a connection between pseudocontinuations and the existence of $((I-\lambda L) \mid \mathcal{M})^{-1}$. Recall from Proposition 1.2 that $\left.\sigma(L \mid \mathcal{M}) \cap \mathbb{D}\right)$ is discrete if and only if the meromorphic function

$$
F(\lambda)=c_{\lambda}(f, g)
$$

if independent of $g$. We will show in Section 7 that if $F$ has finite non-tangential limits on a set $E \subset \mathbb{T}$ of positive measure, then $F$ is a pseudocontinuation of $f$ across $E$. This result will yield several results about the discreteness of $\sigma(L \mid \mathcal{M}) \cap \mathbb{D}$ for certain $L$-invariant subspaces of the Dirichlet spaces.

By the above, the discreteness $\sigma(L \mid \mathcal{M}) \cap \mathbb{D}$ is connected to the index of the corresponding $M_{z}$-invariant subspace $\mathcal{N}$ of the "dual" space which in this case is the unweighted Bergman space $L_{a}^{q}$. For the classical Bergman space $L_{a}^{2}$, the index of an $M_{z}$-invariant subspace $\mathcal{N}$ can be any number in $\mathbb{N} \cup\{\infty\}$, [11] [22] [26] [27] nevertheless, we still have $(1.10)^{9}[7]$. For $f, g \in L_{a}^{q}$, it may be the case that ind $([f, g])=2$ but if these functions are sufficiently "regular" near portions of $\mathbb{T}$, then in fact $\operatorname{ind}([f, g])=1[6]$ [46] [48]. Using our spectral results about certain $L$-invariant subspaces of the $L^{p}$ Dirichlet spaces and the " $H^{2}$-duality", we can prove the following result about $\operatorname{Lat}\left(M_{z}, L_{a}^{q}\right)$.

Corollary 1.15. Let $1<q<+\infty$ and $G, H \in L_{a}^{q}$ such that $G / H$ has a finite non-tangential limit on a set $E \subset \mathbb{T},|E|>0$, then $\operatorname{ind}[G, H]=1$.

${ }^{9}$ Here $[S]$ denotes the smallest $M_{z}$-invariant subspace which contains the set $S$. 


\section{General Banach spaces}

In this section, we list certain elementary observations which follow from our axioms (1.1) through (1.5). Thus throughout this section, $\mathcal{B}$ will be a Banach space of analytic functions on $\mathbb{D}$ which satisfies properties (1.1) through (1.5) and $\mathcal{N}$ will be a non-zero invariant subspace of $L$. The conditions (1.3) and (1.5) imply that $(z-\lambda)^{-1} \in \mathcal{B}$ for all $|\lambda|>1$ and by direct calculation

$$
L\left(\frac{1}{z-\lambda}\right)=\frac{1}{\lambda} \frac{1}{z-\lambda} \text { and } L 1=0 .
$$

Thus $\mathbb{D} \subset \sigma_{p}(L)$, and in fact one checks that each eigenspace is one-dimensional. Also notice by (1.1), (1.2), (1.4), and the closed graph theorem, that the operator

$$
R_{\lambda} f=\frac{z f-\lambda f(\lambda)}{z-\lambda}
$$

is continuous on $\mathcal{B}$ for all $\lambda \in \mathbb{D}$ and a routine computation shows that $(I-\lambda L)^{-1}=R_{\lambda}$. Thus $\sigma(L)=\mathbb{D}^{-}$and it follows that

$$
\sigma(L \mid \mathcal{M}) \subset \mathbb{D}^{-} \quad \forall \mathcal{M} \in \operatorname{Lat}(L, \mathcal{B}) .
$$

Proposition 2.1. For $\mathcal{M} \in \operatorname{Lat}(L, \mathcal{B})$,

$$
\sigma_{\mathrm{ap}}(L \mid \mathcal{M}) \cap \mathbb{D}=\sigma_{p}(L \mid \mathcal{M}) \cap \mathbb{D}=\left\{a \in \mathbb{D}: \frac{1}{1-a z} \in \mathcal{M}\right\} .
$$

Proof. From (2.1) we clearly have

$$
\sigma_{p}(L \mid \mathcal{M}) \cap \mathbb{D}=\left\{a \in \mathbb{D}: \frac{1}{1-a z} \in \mathcal{M}\right\} .
$$

So we just need to verify that $\sigma_{\text {ap }}(L \mid \mathcal{M}) \cap \mathbb{D}=\sigma_{p}(L \mid \mathcal{M}) \cap \mathbb{D}$. Clearly $\sigma_{p}(L \mid \mathcal{M})$ is contained in $\sigma_{\text {ap }}(L \mid \mathcal{M})$. For the other direction, suppose that $\lambda \in \sigma_{\text {ap }}(L \mid \mathcal{M}) \cap \mathbb{D}$. Then, by definition, there is a sequence $\left\{f_{n}\right\} \subset \mathcal{M}$ with

$$
\left\|f_{n}\right\|=1 \quad \text { and } \quad\left\|(L-\lambda I) f_{n}\right\| \rightarrow 0 .
$$

Since $\mathcal{B} \hookrightarrow \operatorname{Hol}(\mathbb{D})$, then $\left\{f_{n}(0)\right\}$ is a bounded sequence of complex numbers and so there is a convergent subsequence (which we also call $\left\{f_{n}(0)\right\}$ ) converging to some $c \in \mathbb{C}$. Since

$$
\left\|\frac{f_{n}-f_{n}(0)}{z}-\lambda f_{n}\right\| \rightarrow 0
$$

and $M_{z}$ is continuous on $\mathcal{B}$, then

$$
\left\|(1-\lambda z) f_{n}-c\right\| \rightarrow 0 .
$$


Since $\sigma\left(M_{z}\right)=\mathbb{D}^{-}$, we conclude

$$
f_{n} \rightarrow \frac{c}{1-\lambda z}
$$

and since $\left\|f_{n}\right\|=1$, then $c \neq 0$. Thus by $(2.1), \lambda \in \sigma_{p}(L \mid \mathcal{M})$.

Proposition 2.2. Let $P=\operatorname{span}_{\mathcal{B}}\left\{z^{n}: n \in \mathbb{N} \cup\{0\}\right\}$. Then the following is true:

(1) $\mathcal{P}=\operatorname{span}_{\mathcal{B}}\left\{(1-\lambda z)^{-1}: \lambda \in \mathbb{D}\right\}$.

(2) If $A \subset \mathbb{D}$ has an accumulation point in $\mathbb{D}$, then

$$
\mathcal{P}=\operatorname{span}_{\mathcal{B}}\left\{(1-\lambda z)^{-1}: \lambda \in A\right\} .
$$

Proof. To prove (1), let

$$
\mathcal{S}=\operatorname{span}_{\mathcal{B}}\left\{\frac{1}{1-\lambda z}: \lambda \in \mathbb{D}\right\} .
$$

To show $\mathcal{S} \subset \mathcal{P}$ we let $\lambda \in \mathbb{D}$ and for $N \in \mathbb{N}$ we consider the polynomial

$$
p_{N}(z)=\sum_{n=0}^{N-1} \lambda^{n} z^{n}
$$

Then

$$
\left\|p_{N}-\frac{1}{1-\lambda z}\right\|=\left\|\frac{\lambda^{N} z^{N}}{1-\lambda z}\right\| \leq|\lambda|^{N}\left\|M_{z}^{N}\right\|\left\|\frac{1}{1-\lambda z}\right\| .
$$

Since the spectral radius of $M_{z} \mid \mathcal{B}$ is equal to one (property (1.5)), then

$$
\lim _{N \rightarrow \infty}\left\|M_{z}^{N}\right\|^{1 / N}=1
$$

and so (2.2) goes to zero as $N \rightarrow \infty$ which shows that $\mathcal{S} \subset \mathcal{P}$.

To show that $\mathcal{P} \subset \mathcal{S}$, we use induction. Note that $(1-\lambda z)^{-1} \in \mathcal{S}$ for all $\lambda \in \mathbb{D}$ and so $1 \in \mathcal{S}$. If $z^{k} \in \mathcal{S} \forall 0 \leq k \leq n$ for some $n \in \mathbb{N} \cup\{0\}$, then for all $\lambda \in \mathbb{D}, \lambda \neq 0$,

$$
\frac{z^{n+1}}{1-\lambda z}=\sum_{k=0}^{n}-\frac{1}{\lambda^{k+1}} z^{n-k}+\frac{1}{\lambda^{n+1}} \frac{1}{1-\lambda z} \in \mathcal{S} .
$$

Furthermore,

$$
\left\|\frac{z^{n+1}}{1-\lambda z}-z^{n+1}\right\| \leq|\lambda|\left\|z^{n+2}\right\|\left\|\left(I-\lambda M_{z}\right)^{-1}\right\| .
$$


But since $\lambda \rightarrow\left(I-\lambda M_{z}\right)^{-1}$ is an (operator valued) analytic function on $\mathbb{D}$, then the right-hand side of (2.3) goes to zero as $|\lambda| \rightarrow 0$. Thus $z^{n+1} \in \mathcal{S}$ and so $\mathcal{P} \subset \mathcal{S}$.

(2) Let $\phi \in \mathcal{B}^{*}$ with

$$
\left\langle\frac{1}{1-\lambda z}, \phi\right\rangle=0 \quad \forall \lambda \in A
$$

Then the function

$$
\lambda \rightarrow\left\langle\frac{1}{1-\lambda z}, \phi\right\rangle
$$

is analytic on $\mathbb{D}$ and (by hypothesis) has zeros which accumulate at some point in $\mathbb{D}$. Hence it must vanish identically on $\mathbb{D}$. Thus $\phi$ annihilates $\mathcal{S}=\mathcal{P}$. An application of the Hahn-Banach theorem completes the proof.

An immediate consequence of Proposition 2.1 and Proposition 2.2 is the following corollary.

Corollary 2.3. For $\mathcal{M} \in \operatorname{Lat}(L, \mathcal{B})$,

(1) $\sigma_{\mathrm{ap}}(L \mid \mathcal{M}) \cap \mathbb{D}=\sigma_{p}(L \mid \mathcal{M}) \cap \mathbb{D}$ is either discrete or all of $\mathbb{D}$.

(2) $\sigma_{\mathrm{ap}}(L \mid \mathcal{M})=\mathbb{D}^{-}$if and only if $\mathcal{M}$ contains all of the polynomials.

Also notice that since $\partial \sigma(L \mid \mathcal{N}) \subset \sigma_{\text {ap }}(L \mid \mathcal{M})$, an elementary argument yields the following dichotomy:

Corollary 2.4. For $\mathcal{M} \in \operatorname{Lat}(L, \mathcal{B})$, either

$$
\sigma(L \mid \mathcal{M}) \cap \mathbb{D}=\sigma_{\text {ap }}(L \mid \mathcal{M}) \cap \mathbb{D}=\sigma_{p}(L \mid \mathcal{M}) \cap \mathbb{D}
$$

and is discrete or $\sigma(L \mid \mathcal{M})=\mathbb{D}^{-}$.

In Section 6 we will show that for the Besov and Dirichlet spaces, it can be the case that $\sigma(L \mid \mathcal{M})=\mathbb{D}^{-}$but $\sigma_{\text {ap }}(L \mid \mathcal{M})=\mathbb{T}$, and $\sigma_{p}(L \mid \mathcal{M}) \cap \mathbb{D}=\emptyset$. We now focus our attention on the part of the spectrum that is contained in $\mathbb{T}$. Before doing so, we make the following remarks.

\section{Remark 2.5.}

(1) To compute $((I-\lambda L) \mid \mathcal{M})^{-1}, \lambda \in \mathbb{C}$ (at least formally) we have

$$
((I-\lambda L) \mid \mathcal{M})^{-1} f=\frac{z f-\lambda c_{\lambda}(f)}{z-\lambda},
$$

where $c_{\lambda}(f)$ is a constant which depends on $f \in \mathcal{M}$.

(2) When $((I-\lambda L) \mid \mathcal{M})^{-1}$ exists, we note from (1.1)

$$
f \rightarrow c_{\lambda}(f)=\left(((I-\lambda L) \mid \mathcal{M})^{-1} f\right)(0)
$$

is a continuous linear functional on $\mathcal{M}$. Moreover the function $\lambda \rightarrow c_{\lambda}(f)$ is analytic for $1 / \lambda$ in the resolvent of $L \mid \mathcal{M}$. 
(3) Since $\sigma(L \mid \mathcal{M}) \subset \mathbb{D}^{-}$, then for $\lambda \in \mathbb{D},((I-\lambda L) \mid \mathcal{M})^{-1}$ exists and $c_{\lambda}(f)=$ $f(\lambda)$ for all $f \in \mathcal{M}$. Moreover, a computation shows that

$$
((I-\lambda L) \mid \mathcal{M})^{-1} L f=\frac{f-f(\lambda)}{z-\lambda} .
$$

This observation will be used many times throughout this paper.

Proposition 2.6. Let $\mathcal{B}$ satisfy (1.1) through (1.5) along with the following additional condition: If $f \in \mathcal{B}$ is analytic in an open neighborhood of a point $\zeta \in \mathbb{T}$, then

$$
\frac{z f-w f(w)}{z-w} \rightarrow \frac{z f-\zeta f(\zeta)}{z-\zeta} \text { in the norm of } \mathcal{B} \text { as } w \rightarrow \zeta(w \in \mathbb{D}) .
$$

Then $\sigma_{\mathrm{ap}}(L \mid \mathcal{M}) \cap \mathbb{T}=\sigma(L \mid \mathcal{M}) \cap \mathbb{T}$ and this equals

$\mathbb{T} \backslash\{1 / \zeta \in \mathbb{T}:$ every $f \in \mathcal{M}$ extends to be analytic in a neighborhood of $\zeta\}$.

Proof. Let

$S=\mathbb{T} \backslash\{1 / \zeta \in \mathbb{T}$ : every $f \in \mathcal{M}$ extends to be analytic in a neighborhood of $\zeta\}$.

Let $\zeta^{-1} \in \mathbb{T}$ with $\zeta^{-1} \notin \sigma_{\text {ap }}(L \mid \mathcal{M})$. Since $\partial \sigma(L \mid \mathcal{M}) \subset \sigma_{\text {ap }}(L \mid \mathcal{M})$, then $\zeta^{-1} \notin \sigma(L \mid \mathcal{M})$. Thus by the remark remark, for each $f \in \mathcal{M}$,

$$
w \rightarrow\left(((I-w L) \mid \mathcal{M})^{-1} f\right)(0)
$$

is an analytic function in a neighborhood of $\zeta$. Also by the above remark,

$$
\left(((I-w L) \mid \mathcal{M})^{-1} f\right)(0)=f(w), \quad|w|<1
$$

and so $f$ has an analytic continuation to a neighborhood of $\zeta$. Hence

$$
\sigma_{\text {ap }}(L \mid \mathcal{M}) \cap \mathbb{T} \supset S .
$$

Now suppose that $\zeta^{-1} \in \mathbb{T}$ such that every $f \in \mathcal{M}$ extends to be analytic near $\zeta$. By the additional condition (2.6) on $\mathcal{B}$ the function

$$
g=\frac{z f-\zeta f(\zeta)}{z-\zeta}
$$

is the norm limit of

$$
g_{n}=\frac{z f-w_{n} f\left(w_{n}\right)}{z-w_{n}} \in \mathcal{M},
$$

for some $w_{n} \in \mathbb{D}$ and $w_{n} \rightarrow \zeta$ and so $g \in \mathcal{M}$. At least formally

$$
((I-\zeta L) \mid \mathcal{M})^{-1} f=\frac{z f-\zeta f(\zeta)}{z-\zeta} .
$$


Suppose that $\left\{f_{n}\right\}$ is a sequence in $\mathcal{M}$ with

$$
f_{n} \rightarrow 0 \quad \text { and } \quad \frac{z f_{n}-\zeta f_{n}(\zeta)}{z-\zeta} \rightarrow h \quad \text { in norm. }
$$

Then by (1.1), equation (2.8) would also hold pointwise in $\mathbb{D}$ and so $f_{n}(\zeta) \rightarrow c$. Thus

$$
h(z)=\frac{-c \zeta}{z-\zeta}
$$

which can only hold when $c=0$ or else $h$ would not be analytic near $\zeta$ which would contradict our choice of $\zeta$. Thus by the closed graph theorem, the righthand-side of (2.7) is a continuous operator on $\mathcal{M}$ and so $\zeta^{-1} \notin \sigma(L \mid \mathcal{M})$.

Remark 2.7. The condition (2.6) seems somewhat mysterious. However, for the main spaces we will be considering, mainly the analytic subspaces of $L^{t}(\mu)$ and $X_{\alpha, p}$, one can easily check, using the dominated convergence theorem and Cauchy's formula, that it indeed holds.

This next result is the primary tool of the paper and will ultimately lead us to the link between pseudocontinuations and the spectrum of $L \mid \mathcal{M}$.

Proposition 2.8. If $|\lambda|>1$ with $1 / \lambda \notin \sigma_{\mathrm{ap}}(L \mid \mathcal{M})$ then $((I-\lambda L) \mid \mathcal{M})^{-1}$ exists if and only if for every $f \in \mathcal{M}$, the quantity

$$
c_{\lambda}(f, \phi)=\left\langle\frac{z f}{z-\lambda}, \phi\right\rangle /\left\langle\frac{\lambda}{z-\lambda}, \phi\right\rangle
$$

is independent of the choice of $\phi \in \mathcal{M}^{\perp}$ with $\left\langle(z-\lambda)^{-1}, \phi\right\rangle \neq 0$. In fact, if $|\lambda|>1$ with $1 / \lambda \notin \sigma(L \mid \mathcal{M})$, then $c_{\lambda}(f, \phi)=c_{\lambda}(f)$ and

$$
((I-\lambda L) \mid \mathcal{M})^{-1} f=\frac{z f-\lambda c_{\lambda}(f)}{z-\lambda}
$$

for all $f \in \mathcal{M}$ and $\phi \in \mathcal{M}^{\perp}$ with $\left\langle(z-\lambda)^{-1}, \phi\right\rangle \neq 0$.

Proof. If $|\lambda|>1$ and $((I-\lambda L) \mid \mathcal{M})^{-1}$ exists. Then by (2.4) for each $f \in \mathcal{M}$ there exists a constant $c_{\lambda}(f) \in \mathbb{C}$ such that

$$
((I-\lambda L) \mid \mathcal{M})^{-1} f=\frac{z f-\lambda c_{\lambda}(f)}{z-\lambda} .
$$

Also note that by (1.2), (1.3), and (1.5), the functions

$$
\frac{z f}{z-\lambda} \text { and } \quad \frac{\lambda c_{\lambda}(f)}{z-\lambda} \in \mathcal{B} \text {. }
$$


Now let $\phi \in \mathcal{M}^{\perp}$ with $\left\langle(z-\lambda)^{-1}, \phi\right\rangle \neq 0$. Notice that $(z-\lambda)^{-1} \notin \mathcal{M}$ and so such $\phi$ 's exist. We have

$$
0=\left\langle((I-\lambda L) \mid \mathcal{M})^{-1} f, \phi\right\rangle=\left\langle\frac{z f}{z-\lambda}, \phi\right\rangle-c_{\lambda}(f)\left\langle\frac{\lambda}{z-\lambda}, \phi\right\rangle
$$

and so

$$
c_{\lambda}(f)=\left\langle\frac{z f}{z-\lambda}, \phi\right\rangle /\left\langle\frac{\lambda}{z-\lambda}, \phi\right\rangle=c_{\lambda}(f, \phi)
$$

is independent of $\phi$.

For the other direction, let $|\lambda|>1$ with $1 / \lambda \notin \sigma_{\text {ap }}(L \mid \mathcal{M})$. Thus, as above, we may choose $\phi \in \mathcal{M}^{\perp}$ such that $\left\langle(z-\lambda)^{-1}, \phi\right\rangle \neq 0$. For $f \in \mathcal{B}$, define

$$
R_{\lambda} f=\frac{z f-\lambda c_{\lambda}(f, \phi)}{z-\lambda}
$$

and note from (2.9) that $R_{\lambda} \mathcal{B} \subset \mathcal{B}$. Furthermore, an application of the closed graph theorem shows that $R_{\lambda}$ is continuous. Since $1 / \lambda \notin \sigma_{\mathrm{ap}}(L \mid \mathcal{M})$, at least formally, $R_{\lambda}$ is an inverse of $(I-\lambda L) \mid \mathcal{M}$. What needs to be shown is that $R_{\lambda} \mathcal{M} \subset \mathcal{M}$. To this end, let $\psi \in \mathcal{M}^{\perp}$ and notice that since $\left\langle(z-\lambda)^{-1}, \phi\right\rangle \neq 0$, then there is a sequence of complex numbers $a_{n} \rightarrow 0$ such that

$$
\left(\psi-a_{n} \phi\right) \mid \mathcal{M}=0 \text { and }\left\langle\frac{1}{z-\lambda}, \psi-a_{n} \phi\right\rangle \neq 0 .
$$

Then

$$
\begin{aligned}
\left\langle R_{\lambda} f, \psi-a_{n} \phi\right\rangle & =\left\langle\frac{z f}{z-\lambda}, \psi-a_{n} \phi\right\rangle-c_{\lambda}(f, \phi)\left\langle\frac{\lambda}{z-\lambda}, \psi-a_{n} \phi\right\rangle \\
& =\left\langle\frac{z f}{z-\lambda}, \psi-a_{n} \phi\right\rangle-c_{\lambda}\left(f, \psi-a_{n} \phi\right)\left\langle\frac{\lambda}{z-\lambda}, \psi-a_{n} \phi\right\rangle \\
& =0
\end{aligned}
$$

since $c_{\lambda}(f, \phi)$ is independent of $\phi$. We now let $a_{n} \rightarrow 0$ and find that $\left\langle R_{\lambda} f, \psi\right\rangle=0$ and so $R_{\lambda} \mathcal{M} \subset \mathcal{M}$.

\section{Cauchy transforms}

Cauchy transforms will play a crucial role in our results. In this section we state some basic properties of Cauchy transforms of measures and prove a generalization of the well-known "jump theorem". In the next section, we will apply our Cauchy transform results to discuss the boundary values of functions in analytic subspaces of $L^{t}(\mu)$. We refer the reader to [16], Chapter 2, Section 3 and [25], Chapter 3 for further information about the Cauchy transform. In this section, we will adhere to the following notation: 


\section{Notation.}

(1) $M_{c}$ is the set of finite, complex, compactly supported Borel measures on $\mathbb{C}$.

(2) $M(K)=\left\{\mu \in M_{c}: \operatorname{supp}(\mu) \subset K\right\}$

(3) $M_{+}(K)=\{\mu \in M(K): \mu \geq 0\}$

For $\lambda \in \mathbb{C}$ and $\mu \in M_{c}$, define the Newtonian potential

$$
U_{\mu}(\lambda)=\int \frac{d|\mu|(z)}{|z-\lambda|} .
$$

By Fubini's theorem, $U_{\mu} \in L_{\text {loc }}^{1}(d A)$ and so

$$
U_{\mu}(\lambda)<+\infty \quad[d A]-\text { a.e. }
$$

Actually, the set where $U_{\mu}$ is infinite is much smaller than a set of area measure zero. It has Newtonian capacity zero, see [25], Chapter 3. Using Fubini's theorem once again, we get that the set

$$
E_{\mu}=\left\{r \in(0, \infty): U_{\mu}(r \zeta) \in L^{1}(\mathbb{T},|d \zeta|)\right\}
$$

has full measure in $\mathbb{R}^{+}$. Moreover, since

$$
\int_{|w|=r} \frac{|d w|}{|w-r \zeta|}=+\infty \quad \forall \zeta \in \mathbb{T}
$$

we see that

$$
r \in E_{\mu} \Rightarrow|\mu|(r \mathbb{T})=0 .
$$

Equation (3.1) allows us (at least $[d A]$-a.e.) to define the Cauchy transform of $\mu$ by

$$
C_{\mu}(\lambda)=\int \frac{d \mu(z)}{z-\lambda}
$$

The Cauchy transform of a measure is clearly analytic off the support of $\mu$. In fact, if the support of $\mu$ is contained in $\mathbb{D}^{-}$, then $C_{\mu} \in H^{p}\left(\mathbb{D}_{e}\right)$ for any $0<p<1$ (see Lemma 3.2 below) and we shall use $C_{\mu}^{+}(\zeta)$ to denote the non-tangential limit values of this function which will exist for $[|d \zeta|]$-a.e. $\zeta \in \mathbb{T}$. We begin with a known result, which is a version of Fatou's classical theorem and is also known as the "jump theorem" (see [21], p. 39).

Proposition 3.1. Let $h \in L^{1}(\mathbb{T},|d \zeta|)$ and let $\sigma \in M(\mathbb{T})$ with $d \sigma \perp|d \zeta|$. Set $d \nu=h|d \zeta|+d \sigma$. Then for each $0<p<1, C_{\nu} \in H^{p}(\mathbb{D})$ (respectively $\left.H^{p}\left(\mathbb{D}_{e}\right)\right)$ and

$$
\lim _{r \rightarrow 1^{-}} C_{\nu}(r \zeta)=C_{\nu}^{+}(\zeta)+2 \pi \bar{\zeta} h(\zeta) \quad[|d \zeta|] \text {-a.e. }
$$


Recall that the "jump theorem" was used in the Introduction to discuss the spectral properties of the backward shift on the Hardy spaces $H^{p}(\mathbb{D}), 1<$ $p<+\infty$. To discuss the backward shift on the analytic subspaces of $L^{t}(\mu)$ for measures that are not always supported in the circle, we will need a more general version of the jump theorem. To this end, we proceed with the following two lemmas.

Lemma 3.2. Let $\mu \in M\left(\mathbb{D}^{-}\right)$. Then for each $0<p<1$, there is a constant $c_{p}>0$ with

$$
\left(\int_{|\lambda|=r}\left|C_{\mu}(\lambda)\right|^{p} \frac{|d \lambda|}{2 \pi r}\right)^{1 / p} \leq \frac{c_{p}}{r}\|\mu\| \quad \forall r \in E_{\mu} .
$$

Proof. First note that by (3.3) $r \in E_{\mu}$ implies that $|\mu|(r \mathbb{T})=0$. Secondly, since $\mu$ can be written as a linear combination of positive measures, we may assume that $\mu \geq 0$.

Let $r \in E_{\mu}$ and define

$$
k_{r}(\lambda)=\int_{|z|>r} \frac{d \mu}{z-\lambda}
$$

Note that $k_{r}$ is defined for all $|\lambda|<r$ and by $(3.2) k_{r}(\lambda)$ is defined for $[|d \zeta|]$-a.e. $|\lambda|=r$.

If $\mu\{|z|>r\}=0$, then $k \equiv 0$ and there is nothing to prove. On the other hand if $\mu\{|z|>r\}>0$, we let

$$
G_{r}(\lambda)=2 \lambda k_{r}(\lambda)+\mu\{|z|>r\}, \quad|\lambda|<r .
$$

A computation reveals that

$$
\Re G_{r}(\lambda)=\int_{|z|>r} \frac{|z|^{2}-|\lambda|^{2}}{|z-\lambda|^{2}} d \mu .
$$

But since $|z|>|\lambda|$, then the above integrand is positive and so $\Re G_{r}(\lambda)>0$ for all $|\lambda|<r$. Moreover, $G_{r}(0)=\mu\{|z|>r\}$. Thus, if

$$
h_{r}(z)=\frac{r+z}{r-z} \mu\{|z|>r\}, \quad|z|<r
$$

then $\Re h_{r}(z)>0$ and $h_{r}(0)=\mu\{|z|>r\}$ which shows that $G_{r}(\lambda)=h_{r}\left(h_{r}^{-1}\left(G_{r}(\lambda)\right)\right.$ is subordinate to $h_{r}$. By Littlewood's subordination theorem [21], p. 10,

$$
\left\|G_{r}\right\|_{H^{p}(r \mathbb{D})} \leq\left\|h_{r}\right\|_{H^{p}(r \mathbb{D})} .
$$


Note here that the norm on $H^{p}(r \mathbb{D})$ is given by

$$
\left(\int_{|z|=r}|f(z)|^{p} \frac{|d z|}{2 \pi r}\right)^{1 / p}
$$

Also notice that

$$
\left\|\frac{r+z}{r-z}\right\|_{H^{p}(r \mathbb{D})}=\left\|\frac{1+z}{1-z}\right\|_{H^{p}(\mathbb{D})}
$$

and so

$$
\left\|G_{r}\right\|_{H^{p}(r \mathbb{D})} \leq \mu\{|z|>r\}\left\|\frac{1+z}{1-z}\right\|_{H^{p}(\mathbb{D})} \leq c_{p}\|\mu\| .
$$

If $s<1$,

$$
k_{r}(s r \zeta)=\int_{|z|>r}\left(\frac{z-r \zeta}{z-s r \zeta}\right) \frac{1}{z-r \zeta} d \mu(z) .
$$

A simple geometric exercise shows that

$$
\frac{|z-r \zeta|}{|z-s r \zeta|} \leq 2, \quad|z|>r, r, s \in[0,1), \zeta \in \mathbb{T}
$$

and so using the fact that $(z-r \zeta)^{-1} d \mu$ is a finite measure for $[|d \zeta|]$-a.e. $\zeta \in \mathbb{T}$ (since $r \in E_{\mu}$ ) along with the dominated convergence theorem shows that

$$
k_{r}(s r \zeta) \rightarrow k_{r}(r \zeta)[|d \zeta|]-\text { a.e. as } s \rightarrow 1^{-} .
$$

By Fatou's Theorem

$$
\int_{\mathbb{T}}\left|\int_{|z|>r} \frac{1}{z-r \zeta} d \mu\right|^{p} \frac{|d \zeta|}{2 \pi} \leq \liminf _{s \rightarrow 1} \int_{\mathbb{T}}\left|k_{r}(s r \zeta)\right|^{p} \frac{|d \zeta|}{2 \pi}=\left\|k_{r}\right\|_{H^{p}(r \mathbb{D})}^{p} .
$$

By (3.5),

$$
\left\|\lambda k_{r}(\lambda)\right\|_{H^{p}(r \mathbb{D})} \leq \frac{1}{2}\left\|G_{r}-\mu\{|z|>r\}\right\|_{H^{p}(r \mathbb{D})} \leq c_{p}\|\mu\| .
$$

Thus

$$
\left\|k_{r}\right\|_{H^{p}(r \mathbb{D})} \leq \frac{1}{r} c_{p}\|\mu\| .
$$

In a very similar way

$$
\left\|\int_{|z|<r} \frac{d \mu}{z-\lambda}\right\|_{H^{p}(|\lambda|>r)} \leq \frac{1}{r} c_{p}\|\mu\|
$$

and thus the proof is complete. 
Lemma 3.3. Let $\mu \in M\left(\mathbb{D}^{-}\right)$with $|\mu|(\mathbb{T})=0$. Then for all $0<p<1$,

$$
\int_{|\zeta|=1}\left|C_{\mu}(r \zeta)-C_{\mu}\left(\frac{1}{r} \zeta\right)\right|^{p}|d \zeta| \rightarrow 0, \quad \text { as } r \rightarrow 1^{-}, r \in E_{\mu} .
$$

Proof. Let $\varepsilon>0$ be given and choose $\delta>0$ with $|\mu|\{1-\delta \leq|z|<1\}<\varepsilon$. Then we can write $\mu$ as

$$
\mu=\left.\mu\right|_{\{1-\delta \leq|z|<1\}}+\left.\mu\right|_{\{|z|<1-\delta\}}=\mu_{1}+\mu_{2} .
$$

For $0<p<1$ and $r \in E_{\mu} \cap(0,1)$, we have

$$
\begin{aligned}
\int_{|\zeta|=1}\left|C_{\mu}(r \zeta)-C_{\mu}\left(\frac{1}{r} \zeta\right)\right|^{p}|d \zeta| \leq \int_{|\zeta|=1} \mid & C_{\mu_{1}}(r \zeta)-\left.C_{\mu_{1}}\left(\frac{1}{r} \zeta\right)\right|^{p}|d \zeta| \\
& +\int_{|\zeta|=1}\left|C_{\mu_{2}}(r \zeta)-C_{\mu_{2}}\left(\frac{1}{r} \zeta\right)\right|^{p}|d \zeta| .
\end{aligned}
$$

Since $C_{\mu_{2}}$ is continuous near $\mathbb{T}$, then the second integral above converges to zero as $r \rightarrow 1^{-}$. By Lemma 3.2, the first integral is bounded by $c_{p} \varepsilon^{p}$.

For $\mu \in M\left(\mathbb{D}^{-}\right)$, write $\mu=\mu|\mathbb{D}+\mu| \mathbb{T}$ and use the basic fact that convergence in $L^{p}(\mathbb{T},|d \zeta|)$ implies convergence in measure along with Proposition 3.1 and Lemma 3.3, to get the following generalization of the "jump theorem".

Corollary 3.4. Let $\mu \in M\left(\mathbb{D}^{-}\right)$. Then

$$
C_{\mu}(r \zeta) \rightarrow C_{\mu}^{+}(\zeta)+2 \pi \bar{\zeta} \frac{d \mu \mid \mathbb{T}}{|d \zeta|}(\zeta) \quad \text { in measure }[|d \zeta|] \text { as } r \rightarrow 1^{-}, r \in E_{\mu}
$$

We would like to mention at this point that instead of Lemma 3.2 and Lemma 3.3 we also could have deduced Corollary 3.4 from the weak-type estimate

$$
\left|\left\{\zeta \in \mathbb{T}:\left|C_{\mu}(r \zeta)\right|>\lambda\right\}\right| \leq \frac{C}{r} \frac{\|\mu\|}{\lambda}, \quad r \in E_{\mu} .
$$

To prove this estimate, one can proceed as follows: For fixed $r \in E_{\mu}$, one writes $\mu=\mu_{1}+\mu_{2}$, where $\mu_{1}(\{|z|>r\})=0$ and $\mu_{2}(\{|z|<r\})=0$ (note that $|\mu|(r \mathbb{T})=0$ by (3.3)). Then one can apply the standard weak-type estimates for Cauchy transforms of measures [40], Theorem 6.2.2, to the sweeps of $\mu_{1}$ and $\mu_{2}$ to $r \mathbb{T}$. We omit the details.

\section{Analytic subspaces of $L^{t}(\mu)$}

In this section, we will first define the analytic subspaces of $L^{t}(\mu)$ and then prove our main theorem about the spectral properties of $\operatorname{Lat}(L, \mathcal{B})$. 
4.1. Definition and basic properties. Let $\mathcal{B}$ be a Banach space of analytic functions on $\mathbb{D}$ which satisfies properties (1.1) through (1.5), $1 \leq t<+\infty$, and $\mu \in M_{c}, \mu \neq 0$. Furthermore, suppose there is a linear isometry

$$
U: \mathcal{B} \rightarrow L^{t}(\mu)
$$

such that

$$
U 1=1 \quad \text { and } \quad U\left(M_{z} \mid \mathcal{B}\right)=\left(M_{z} \mid L^{t}(\mu)\right) U
$$

For now, we will denote the range of $U$ by $\mathcal{E}$. As mentioned in Section 1 , we shall later identify $\mathcal{B}$ and $\mathcal{E}$. It is clear that $\mathcal{E}$ is $M_{z}$-invariant and since $1 \in \mathcal{E}$, it follows that $P^{t}(\mu) \subset \mathcal{E}$, where $P^{t}(\mu)$ is the closure of the analytic polynomials in $L^{t}(\mu)$. Furthermore, since $\sigma\left(M_{z} \mid \mathcal{B}\right)=\mathbb{D}^{-}$, one argues that the support of $\mu$ is contained in $\mathbb{D}^{-}$.

Proposition 4.1. With $\mu$ as above, $d \mu|\mathbb{T} \ll| d \zeta \mid$.

Proof. Suppose to the contrary. Then $d \mu|\mathbb{T}=g| d \zeta \mid+d \sigma$ for some nonzero $\sigma \perp|d \zeta|$. By [24], p. 126, problem 2, there is a sequence of analytic polynomials $\left\{p_{n}\right\}$ which converge in $L^{t}(\mu)$ to a non-zero function $h \in P^{t}(\mu) \subset \mathcal{E}$ with $h \mid \mathbb{D}=0$ and $h|\mathbb{T}=0| d \zeta \mid$-a.e. Letting $[h]$ denote the smallest $M_{z}$-invariant subspace of $\mathcal{E}$ which contains $h$ we see that since $|h|^{t} d \mu=|h|^{t} d \sigma \perp|d \zeta|$ then $P^{t}\left(|h|^{t} d \mu\right)=L^{t}\left(|h|^{t} d \mu\right)$ and so

$$
0 \notin \sigma\left(M_{z} \mid[h]\right) .
$$

Since $U\left(M_{z} \mid \mathcal{B}\right)=\left(M_{z} \mid \mathcal{E}\right) U$, then

$$
(0) \neq \mathcal{M}=U^{-1}[h] \in \operatorname{Lat}\left(M_{z}, \mathcal{B}\right) .
$$

By (1.1), $z \mathcal{M} \neq \mathcal{M}$ and so $0 \in \sigma\left(M_{z} \mid \mathcal{M}\right)$ which is a contradiction.

Proposition 4.2. Let $\mathcal{B}, \mathcal{E}$, and $\mu$ be as above. Set

$$
g=\frac{d \mu \mid \mathbb{T}}{|d \zeta|} \quad \text { and } \quad S_{g}=\mathbb{T} \backslash\{\zeta \in \mathbb{T}: g(\zeta)=0\} .
$$

For each $f \in \mathcal{B}$ and $0<r<1$, write $f_{r}(z)=f(r z), z \in \mathbb{D}^{-}$. Then for every $f \in \mathcal{B}$ we have

(1) $(U f)(z)=f(z)$ for $[\mu]$-a.e. $z \in \mathbb{D}$.

(2) $f_{r}\left|S_{g} \rightarrow(U f)\right| S_{g}$ in measure $[|d \zeta|]$ as $r \rightarrow 1^{-}$.

Before we proceed to the proof, we record the following elementary fact which we will use several times throughout this section. 
Lemma 4.3. Let $f_{n}, g_{n}$, and $h_{n}(n \in \mathbb{N})$ be $[|d \zeta|]$-measurable functions on $\mathbb{T}$ with $h_{n}=f_{n} g_{n}$ a.e.,$h_{n} \rightarrow h$ and $g_{n} \rightarrow g$ in measure, $g \neq 0$ on some set $A$ of positive measure. Then $f_{n}|A \rightarrow h / g| A$ in measure.

Proof of Proposition 4.2. Define an operator $L_{\varepsilon}$ on $\mathcal{E}$ by

$$
L_{\varepsilon}=U L U^{-1}
$$

and note that for $f \in \mathcal{B}$

$$
\left(M_{z} \mid \mathcal{E}\right) U L f=U\left(M_{z} \mid \mathcal{B}\right) L f=U f-f(0) U 1=U f-f(0)
$$

and hence

$$
\left(L_{\varepsilon} U f\right)(z)=\frac{(U f)(z)-f(0)}{z}, \quad[\mu]-\text { a.e. } z \neq 0 .
$$

Furthermore, one proves by induction that for $n \in \mathbb{N}$

$$
\left.L_{\mathcal{E}}^{n}(U f)\right)(z)=\frac{1}{z^{n}}\left((U f)(z)-\sum_{k=0}^{n-1} \frac{f^{(k)}(0)}{k !} z^{k}\right) \quad[\mu]-\text { a.e., } z \neq 0 .
$$

To prove (1) we fix $f \in \mathcal{B}$. For $0<R<1$ choose $\varepsilon>0$ so that $R(1+\varepsilon)<1$ and let $N \in \mathbb{N}$ such that $\left\|L_{\mathcal{E}}^{n}\right\| \leq(1+\varepsilon)^{n}$ for all $n \geq N$. This can indeed be done since the spectral radius of $L_{\mathcal{\varepsilon}}$ is one. Then

$$
\begin{aligned}
& \int_{R \mathbb{D} \backslash\{0\}}\left|(U f)(z)-\sum_{k=0}^{n-1} \frac{f^{(k)}(0)}{k !} z^{k}\right|^{t} d \mu \\
\leq & R^{n t} \int_{R \mathbb{D} \backslash\{0\}}\left|\frac{1}{z^{n}}\left((U f)(z)-\sum_{k=0}^{n-1} \frac{f^{(k)}(0)}{k !} z^{k}\right)\right|^{t} d \mu \\
\leq & R^{n t}\left\|L_{\mathcal{E}}^{n} U f\right\|^{t} \\
\leq & R^{n t}(1+\varepsilon)^{n t}\|U f\|^{t}
\end{aligned}
$$

which goes to zero as $n \rightarrow \infty$. Hence $(U f)(z)=f(z)$ for $[\mu]$-a.e. $|z|<R$ $(z \neq 0)$, hence for $[\mu]$-a.e. $|z|<1(z \neq 0)$. Furthermore, if $\mu\{0\}>0$, then by $(4.2)(U f)(0)=f(0)$ and hence we have shown (1).

To prove (2), let $s$ be the conjugate index to $t$, so $\mathcal{E}^{\perp} \subset L^{s}(\mu)$. Fix $h \in \mathcal{E}^{\perp}$ and write (using the notation (3.2))

$$
E=E_{(U f) h d \mu} \cap E_{h d \mu} \cap E_{d \mu}
$$


and notice this set has full measure in $[0,1]$. We note that for every $\lambda \in \mathbb{D}$, $|\lambda| \in E$, we have

$$
\left(\left(I-\lambda L_{\mathcal{E}}\right)^{-1} L_{\mathcal{E}} U f\right)(z)=\frac{(U f)(z)-f(\lambda)}{z-\lambda}, \quad[\mu]-\text { a.e. } z \in \mathbb{D}^{-} .
$$

Indeed for $f \in \mathcal{B}$

$$
\begin{aligned}
\left(M_{z-\lambda} \mid \mathcal{E}\right)\left(I-\lambda L_{\mathcal{E}}\right)^{-1} L_{\mathcal{E}} U f & =U\left(M_{z-\lambda} \mid \mathcal{B}\right)(I-\lambda L)^{-1} L f & & \\
& =U(f-f(\lambda)) & & \text { by }(2.5) \\
& =U f-f(\lambda) & & \text { since } U 1=1 .
\end{aligned}
$$

Hence since $|\lambda| \in E$, then $\mu(|\lambda| \mathbb{T})=0$ and so

$$
\left(\left(I-\lambda L_{\varepsilon}\right)^{-1} L_{\varepsilon} U f\right)(z)=\frac{(U f)(z)-f(\lambda)}{z-\lambda}, \quad[\mu]-\text { a.e. } z \in \mathbb{D}^{-} .
$$

From this, we conclude that

$$
\int \frac{(U f)(z)-f(r \zeta)}{z-r \zeta} \bar{h}(z) d \mu(z)=0, \quad \text { for all } r \in E, \zeta \in \mathbb{T} \text {, and } h \in \mathcal{E}^{\perp} .
$$

This implies

$$
C_{\mu}((U f) \bar{h})(r \zeta)=f(r \zeta) C_{\mu}(\bar{h})(r \zeta), \quad \text { for all } r \in E \text { and }[|d \zeta|] \text {-a.e. } \zeta \in \mathbb{T} .
$$

Here we have used $C_{\mu}(F)(w)$ to denote the Cauchy transform

$$
C_{\mu}(F)(w)=\int \frac{F(z)}{z-w} d \mu(z), \quad F \in L^{1}(\mu) .
$$

Next we note that by (1.5) for $f \in \mathcal{B}$ and $|\lambda|>1, f /(z-\lambda) \in \mathcal{B}$ and so $k=U(f /(z-\lambda)) \in \mathcal{E}$. By $(4.1)(z-\lambda) k=U f$ and so $k=(U f) /(z-\lambda)$ belongs to $\mathcal{E}$. Thus (applying the above first with a general $f$ and then with $f=1 \in \mathcal{B}$ by $(1.3))$

$$
C_{\mu}((U f) \bar{h})(\lambda)=C_{\mu}(\bar{h})(\lambda)=0 \quad \forall|\lambda|>1 .
$$

Hence it follows from Corollary 3.4 that

$$
C_{\mu}(\bar{h})(r \zeta) \rightarrow 2 \pi \bar{\zeta} \overline{h(\zeta)} g(\zeta),
$$

and

$$
C_{\mu}((U f) \bar{h})(r \zeta) \rightarrow 2 \pi \bar{\zeta} \overline{h(\zeta)} g(\zeta)(U f)(\zeta) \quad \text { in measure }[|d \zeta|] \text { as } r \rightarrow 1^{-}, r \in E \text {. }
$$

Thus by (4.3) and Lemma 4.3, we see that on the set

$$
S_{h g}=S_{g} \backslash\{\zeta \in \mathbb{T}: h(\zeta)=0\}
$$


$f_{r}(\zeta)$ converges in measure $[|d \zeta|]$ to $(U f)(\zeta)$ as $r \rightarrow 1^{-}, r \in E$. But $f$ is continuous on $\mathbb{D}$ and so it is an easy exercise to show that

$$
f_{r}\left|S_{h g} \rightarrow(U f)\right| S_{h g} \quad \text { in measure }[|d \zeta|] \text { as } r \rightarrow 1^{-} .
$$

We shall conclude the proof by showing that there is an $h \in \mathcal{E}^{\perp}$ such that $S_{g} \backslash S_{h g}$ has Lebesgue measure zero. Let

$$
\mathcal{C}=\left\{h \in \mathcal{E}^{\perp}:\|h\|_{L^{s}(\mu)} \leq 1\right\}
$$

and notice that $\mathcal{C}$ is a closed, convex, and bounded subset of $L^{s}(\mu)$. By a result of Chaumat [16], p. 246, there is an $h_{0} \in \mathcal{C}$ such that

$$
|h| d \mu \ll\left|h_{0}\right| d \mu \quad \forall h \in \mathcal{C} .
$$

We claim that $S_{g} \backslash S_{h_{0} g}$ has Lebesgue measure zero. Indeed, suppose that $S_{g} \backslash S_{h_{0} g}$ had positive Lebesgue measure. Then since $d \mu|\mathbb{T}=g| d \zeta \mid$, then $\mu\left(S_{g} \backslash S_{h_{0} g}\right)>0$ and there would be a compact subset $F \subset S_{g} \backslash S_{h_{0} g}$ such that $0 \neq \chi_{F} \neq 1$ in $L^{t}(\mu \mid \mathbb{T})$. But then from (4.4) we get $h \mid F=0[\mu]$-a.e. for each $h \in \mathcal{C}$ and so $h \mid F=0[\mu]$-a.e. for each $h \in \mathcal{E}^{\perp}$. This implies $\chi_{F} \in \mathcal{E}$. Now argue as in Proposition 4.1 to derive a contradiction.

\section{Remark 4.4.}

(1) As in the proof above, one notes that for $\lambda \in \mathbb{D}$

$$
\left(\left(I-\lambda L_{\varepsilon}\right)^{-1} L_{\varepsilon} U f\right)(z)=\frac{(U f)(z)-f(\lambda)}{z-\lambda}, \quad[\mu] \text {-a.e. } z \in \mathbb{D}^{-}, z \neq \lambda .
$$

If $\mu\{\lambda\}>0$, then one verifies that

$$
\left(\left(I-\lambda L_{\varepsilon}\right)^{-1} L_{\mathcal{E}} U f\right)(\lambda)=f^{\prime}(\lambda) .
$$

We will not have to use this last observation in our analysis below because, as was done in the above proof, we will always choose $\lambda \in \mathbb{D}$ so that $|\lambda| \in E_{d \mu}$.

(2) As we mentioned in Section 1, we shall now suppress the isometry $U$ in our notation and identify the spaces $\mathcal{B}$ and $\mathcal{E}$ and the operators $L$ and $L_{\mathcal{E}}$ and call $\mathcal{B}$ an analytic subspace of $L^{t}(\mu)$.

(3) We note that if $\phi \in H^{\infty}(\mathbb{D})$ and $f \in \mathcal{B}$, then it follows easily from the dominated convergence theorem, Fatou's theorem, and the fact that $d \mu|\mathbb{T} \ll| d \zeta \mid$, that

$$
\phi_{r} f \rightarrow \phi f \quad \text { in } \mathcal{B} \text {. }
$$

Hence any $M_{z}$-invariant subspace of $\mathcal{B}$ is invariant under multiplication by all $H^{\infty}(\mathbb{D})$ functions and in particular $H^{\infty}(\mathbb{D}) \subset P^{t}(\mu) \cap L^{\infty}(\mu) \subset \mathcal{B}$. These observations also follow from a part of a special case of Thomson's 
theorem [45], which implies that under our hypothesis, for any nonzero $f \in \mathcal{B}, H^{\infty}(\mathbb{D})$ is isometrically and isomorphically and weak-* homeomorphic to $P^{t}\left(|f|^{t} d \mu\right) \cap L^{\infty}\left(|f|^{t} d \mu\right)$.

(4) We also mention that part 2 of Proposition 4.2 implies (in certain settings) a result of Olin and Yang [34] [49] ${ }^{10}$.

4.2. L-invariant subspaces in analytic subspaces. In this section, we shall combine the results of the previous two sections and prove our main theorems about $L$-invariant subspaces of analytic subspaces of $L^{t}(\mu)$.

Let $\mathcal{B}$ be an analytic subspace of $L^{t}(\mu)$ of the type considered in the previous section. For $f \in \mathcal{B}$, we let $[f]$ denote the smallest $M_{z}$-invariant subspace of $\mathcal{B}$ that contains $f$, i.e., $[f]$ is the $L^{t}(\mu)$-closure of $H^{\infty}(\mathbb{D}) f$. Notice also that if $h \in L^{s}(\mu)$, then $C_{\mu}(f \bar{h}) \equiv 0$ on $\mathbb{D}_{e}$ if and only if $h \in[f]^{\perp}$.

Lemma 4.5. Let $\mathcal{M} \in \operatorname{Lat}(L, \mathcal{B})$. If $f_{0} \in \mathcal{B}$ with $L f_{0} \in \mathcal{M}$, and $\left[f_{0}\right] \not \subset \mathcal{M}$, then for each $f \in \mathcal{M}$, there exists a unique meromorphic function $g_{f} \in \mathfrak{N}\left(\mathbb{D}_{e}\right)$ such that

$$
\left(f / f_{0}\right)_{r} \rightarrow g_{f} \quad \text { in measure }[|d \zeta|] \text { on } \mathbb{T} \text { as } r \rightarrow 1^{-} .
$$

Furthermore, the identity

$$
\int \frac{f \bar{h}}{z-\lambda} d \mu=g_{f}(\lambda) \int \frac{f_{0} \bar{h}}{z-\lambda} d \mu
$$

holds for all $\lambda \in \mathbb{D}_{e}$ and $h \in \mathcal{M}^{\perp}$.

Proof. Let $h_{0} \in \mathcal{M}^{\perp} \backslash\left[f_{0}\right]^{\perp} \subset L^{s}(\mu)$, where $s$ is a conjugate index to $t$. Then $C_{\mu}\left(f_{0} \overline{h_{0}}\right)$ is not identically zero in $\mathbb{D}_{e}$ and so by Lemma 3.2 , for $f \in \mathcal{M}$, the function

$$
g_{f}(\lambda)=\frac{C_{\mu}\left(f \overline{h_{0}}\right)(\lambda)}{C_{\mu}\left(f_{0} \overline{h_{0}}\right)(\lambda)}
$$

defines a meromorphic function in $\mathfrak{N}\left(\mathbb{D}_{e}\right)$ (since it is the quotient of two $H^{p}\left(\mathbb{D}_{e}\right)$ functions).

For $f \in \mathcal{B}$

$$
\frac{f-\left(f / f_{0}\right)(\lambda) f_{0}}{z-\lambda}=(I-\lambda L)^{-1} L f-\frac{f}{f_{0}}(\lambda)(I-\lambda L)^{-1} L f_{0}
$$

which, by our hypothesis, belongs to $\mathcal{M}$ whenever $f \in \mathcal{M}$ and $|\lambda|<1$ with $f_{0}(\lambda) \neq 0$, see (2.5). As in the proof of Proposition 4.2 the above also holds pointwise for $[\mu]$-a.e. $z \in \mathbb{D}^{-}$whenever $|\lambda| \in E_{d \mu}$. Let

$$
P=\left\{r \in[0,1): f / f_{0} \text { has a pole on } r \mathbb{T}\right\}
$$

10 Actually, their result is in a more general setting and works for domains other than the disk. The hypothesis of their result also depends on the local behavior of the function $f$. 
and note that $P$ is a countable set. For $|\lambda| \in E_{d \mu} \backslash P$, it follows that

$$
\int \frac{f-\left(f / f_{0}\right)(\lambda) f_{0}}{z-\lambda} \bar{h} d \mu=0
$$

for each $f \in \mathcal{M}, h \in \mathcal{M}^{\perp}$. Set

$$
E=E_{f \overline{h_{0}}} d \mu \cap E_{f_{0} \overline{h_{0}} d \mu} \cap E_{d \mu},
$$

where $E_{\mu}$ was defined as in (3.2) and note that for $r \in E \backslash P$ equation (4.6) says

$$
C_{\mu}\left(f \overline{h_{0}}\right)(r \zeta)=\frac{f}{f_{0}}(r \zeta) C_{\mu}\left(f_{0} \overline{h_{0}}\right)(r \zeta) \quad[|d \zeta|] \text {-a.e. }
$$

Now fix $f \in \mathcal{M}$, write $d \mu=d \mu_{1}+g|d \zeta|$ (where $\mu_{1}(\mathbb{T})=0, g \in L^{1}(\mathbb{T},|d \zeta|)$, and $g \geq 0$. Recall that $d \mu|\mathbb{T} \ll| d \zeta \mid$.), and let

$$
S_{g}=\mathbb{T} \backslash\{\zeta \in \mathbb{T}: g(\zeta)=0\} .
$$

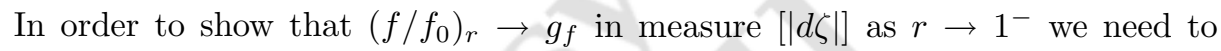
consider two cases: $S_{g}$ and $\mathbb{T} \backslash S_{g}$.

On the set $S_{g}$, it follows from Proposition 4.2 that

$$
\left(f_{0}\right)_{r} \rightarrow f_{0} \text { and } f_{r} \rightarrow f \quad \text { in measure. }
$$

We now multiply (4.7) by $f_{0}(r \zeta), r \in E \backslash P$, and apply Corollary 3.4 and Proposition 4.2 to obtain

$$
\begin{gathered}
f_{0}(\zeta)\left(C_{\mu}^{+}\left(f \overline{h_{0}}\right)(\zeta)+2 \pi \bar{\zeta} f(\zeta) \overline{h_{0}}(\zeta) g(\zeta)\right) \\
-f(\zeta)\left(C_{\mu}^{+}\left(f_{0} \overline{h_{0}}\right)(\zeta)+2 \pi \bar{\zeta} f_{0}(\zeta) \overline{h_{0}}(\zeta) g(\zeta)\right)
\end{gathered}
$$

which is equal to zero for $[|d \zeta|]$-a.e. $\zeta \in S_{g}$. In particular, it follows that

$$
f_{0}(\zeta) C_{\mu}^{+}\left(f \overline{h_{0}}\right)(\zeta)-f(\zeta) C_{\mu}^{+}\left(f_{0} \overline{h_{0}}\right)(\zeta)=0
$$

for $[|d \zeta|]$-a.e. $\zeta \in S_{g}$. Now recall the definition of $g_{f}$ and the fact that $h_{0}$ was chosen so that

$$
C_{\mu}^{+}\left(f_{0} \overline{h_{0}}\right)(\zeta) \neq 0 \quad[|d \zeta|]-\text { a.e. }
$$

and hence

$$
f_{0}(\zeta) g_{f}(\zeta)-f(\zeta)=0 \quad[|d \zeta|] \text {-a.e., } \zeta \in S_{g}
$$


This implies, by Lemma 4.3, that $\left(f / f_{0}\right)_{r} \rightarrow g_{f}$ in measure as $r \rightarrow 1^{-}$on the set $\left\{\zeta \in S_{g}: f_{0}(\zeta) \neq 0\right\}$. If $f_{0}(\zeta)=0$ on a set $S \subset S_{g}$ of positive Lebesgue measure, then by $(4.9) f(\zeta)=0[|d \zeta|]$-a.e. on $S$ and by Corollary 3.4

$$
C_{\mu}\left(f \overline{h_{0}}\right)(r \zeta) \rightarrow C_{\mu}^{+}\left(f \overline{h_{0}}\right)(\zeta)
$$

$$
C_{\mu}\left(f_{0} \overline{h_{0}}\right)(r \zeta) \rightarrow C_{\mu}^{+}\left(f_{0} \overline{h_{0}}\right)(\zeta)
$$

in measure on $S$ as $r \rightarrow 1^{-}, r \in E \backslash P$. Using (4.7), Lemma 4.3, and (4.8), we get that

$$
\left(\frac{f}{f_{0}}\right)_{r}\left|S \rightarrow \frac{C_{\mu}^{+}\left(f \overline{h_{0}}\right)}{C_{\mu}^{+}\left(f_{0} \overline{h_{0}}\right)}\right| S=g_{f} \mid S \quad \text { in measure as } r \rightarrow 1^{-}, r \in E \backslash P \text {. }
$$

This shows

$$
\left(\frac{f}{f_{0}}\right)_{r}\left|S_{g} \rightarrow g_{f}\right| S_{g} \quad \text { in measure as } r \rightarrow 1^{-}, r \in E \backslash P \text {. }
$$

On the set $\mathbb{T} \backslash S_{g}$, we apply Corollary 3.4 again to get that equations (4.10) and (4.11) hold (this time in measure on $\mathbb{T} \backslash S_{g}$ as $r \rightarrow 1^{-}, r \in E$ ) and thus using (4.7), (4.8), and Lemma 4.3, we get that

$$
\left(\frac{f}{f_{0}}\right)_{r}\left|\mathbb{T} \backslash S_{g} \rightarrow g_{f}\right| \mathbb{T} \backslash S_{g} \quad \text { in measure as } r \rightarrow 1^{-}, r \in E \backslash P \text {. }
$$

Finally, since $f / f_{0}$ is meromorphic in $\mathbb{D}$, it is an easy exercise, which we leave to the reader, to show that $\left(f / f_{0}\right)_{r} \rightarrow g_{f}$ in measure as $r \rightarrow 1^{-}$.

Of course, the limit in measure of $f / f_{0}$ is unique and $\mathfrak{N}\left(\mathbb{D}_{e}\right)$ functions are uniquely determined by their boundary values, hence $g_{f}$ is unique.

To prove the identity (4.5) we let $h \in \mathcal{M}^{\perp}$ be arbitrary and define a meromorphic function on $\mathbb{D}_{e}$ by

$$
F(\lambda)=C_{\mu}(f \bar{h})(\lambda)-g_{f}(\lambda) C_{\mu}\left(f_{0} \bar{h}\right)(\lambda)
$$

By our previous work, $F \in \mathfrak{N}\left(\mathbb{D}_{e}\right)$ and it suffices to prove (by Privalov's uniqueness theorem) that the non-tangential limit function

$$
F(\zeta)=C_{\mu}^{+}(f \bar{h})(\zeta)-g_{f}(\zeta) C_{\mu}^{+}\left(f_{0} \bar{h}\right)(\zeta)
$$


is zero $[|d \zeta|]$-a.e. on $\mathbb{T}$. We take the limit in measure as $r \rightarrow 1^{-}, r \in E \backslash P$, of (4.7) (this time with $h_{0}=h$ and $E$ suitably changed) and apply Corollary 3.4 and (1) to obtain

$$
\begin{aligned}
0 & =C_{\mu}^{+}(f \bar{h})(\zeta)+2 \pi \bar{\zeta} f(\zeta) \bar{h}(\zeta) g(\zeta)-g_{f}(\zeta)\left(C_{\mu}^{+}\left(f_{0} \bar{h}\right)(\zeta)+2 \pi \bar{\zeta} f_{0}(\zeta) \bar{h}(\zeta) g(\zeta)\right) \\
& =F(\zeta)+2 \pi \overline{\zeta h}(\zeta) g(\zeta)\left(f(\zeta)-g_{f}(\zeta) f_{0}(\zeta)\right) \quad[|d \zeta|]-\text { a.e. }
\end{aligned}
$$

But by (4.9) we have

$$
g(\zeta)\left(f(\zeta)-g_{f}(\zeta) f_{0}(\zeta)\right)=0 \quad[|d \zeta|] \text {-a.e. }
$$

which shows that $F \equiv 0$ as desired.

We are now ready to prove the main theorem of this section.

Theorem 4.6. Let $1 \leq t<+\infty$ and let $\mu$ be a non-trivial finite Borel measure on $\mathbb{D}^{-}$such that the space $\mathcal{B}$ is an analytic subspace of $L^{t}(\mu)$. Let $\mathcal{M} \in \operatorname{Lat}(L, \mathcal{B})$ with $\mathcal{M} \neq(0)$. Then:

(1) $\sigma(L \mid \mathcal{M})=\sigma_{\mathrm{ap}}(L \mid \mathcal{M})$. More precisely,

(a) $\sigma(L \mid \mathcal{M}) \cap \mathbb{D}=\sigma_{\text {ap }}(L \mid \mathcal{M}) \cap \mathbb{D}=\sigma_{p}(L \mid \mathcal{M}) \cap \mathbb{D}=\left\{a \in \mathbb{D}:(1-a z)^{-1} \in\right.$ $\mathcal{N}\}$.

(b) $\sigma(L \mid \mathcal{M}) \cap \mathbb{T}=\sigma_{\text {ap }}(L \mid \mathcal{M}) \cap \mathbb{T}$ and is the following set

$\mathbb{T} \backslash\{1 / \zeta \in \mathbb{T}$ : every $f \in \mathcal{M}$ extends to be analytic in a neighborhood of $\zeta\}$.

(2) $\sigma(L \mid \mathcal{M})=\mathbb{D}^{-}$if and only if $\mathcal{M}$ contains all of the polynomials.

(3) If $\mathcal{M}$ does not contain all of the polynomials, then $\sigma(L \mid \mathcal{M}) \cap \mathbb{D}$ is a Blaschke sequence. Furthermore, for each $f \in \mathcal{M}$ there exists a unique $\tilde{f} \in \mathfrak{N}\left(\mathbb{D}_{e}\right)$ such that

$$
f_{r}|\mathbb{T} \rightarrow \tilde{f}| \mathbb{T}^{11} \text { in measure }[|d \zeta|] \text { as } r \rightarrow 1^{-} .
$$

Moreover, the function $\tilde{f}$ is given by

$$
\tilde{f}(\lambda)=\int \frac{f \bar{h}}{z-\lambda} d \mu / \int \frac{\bar{h}}{z-\lambda} d \mu
$$

for all $h \in \mathcal{M}^{\perp}$ which do not annihilate all of the polynomials.

11 Here we mean the non-tangential limit values of the Nevanlinna function $\tilde{f}$ on $\mathbb{T}$. 
Proof.

(1) If $\mathcal{M}$ contains all of the polynomials, then by Proposition 2.1 and Proposition $2.2, \sigma_{\text {ap }}(L \mid \mathcal{M})=\mathbb{D}^{-}$and so

$$
\mathbb{D}^{-}=\sigma_{\mathrm{ap}}(L \mid \mathcal{M})=\sigma(L \mid \mathcal{M}) .
$$

If $\mathcal{M}$ does not contain all polynomials, then apply Lemma 4.5 with $f_{0}=1$ along with Proposition 2.8 and Proposition 2.1. This proves (a). An application of the dominated convergence theorem and the Cauchy formula shows that $\mathcal{B}$ satisfies the hypothesis of Proposition 2.6, and thus (b) follows now from Proposition 2.6.

(2) Use (1) and Corollary 2.3.

(3) If $\mathcal{M}$ does not contain all polynomials, then there is an $h \in \mathcal{M}^{\perp}$ such that $C_{\mu}(\bar{h}) \not \equiv 0$ in $\mathbb{D}_{e}$. By (1) we have

$$
\sigma(L \mid \mathcal{M}) \cap \mathbb{D}=\left\{a \in \mathbb{D}: \frac{1}{1-a z} \in \mathcal{M}\right\} \subset\left\{a \in \mathbb{D}: \int \frac{\bar{h}}{1-a z} d \mu=0\right\}
$$

and since $C_{\mu}(\bar{h}) \in H^{p}\left(\mathbb{D}_{e}\right)$, the points of this set form a Blaschke sequence. Finally the rest of the proof of (3) follows from Lemma 4.5 with $f_{0}=1$.

We saw in the previous theorem that $\sigma(L \mid \mathcal{M})=\mathbb{D}^{-}$if and only if $\mathcal{M}$ contains all of the polynomials. We will now show that for certain measures, this condition is also equivalent to $\mathcal{N}$ being $M_{z}$-invariant.

Corollary 4.7. Let $1 \leq t<+\infty$ and let $\mu$ be a non-trivial finite Borel measure on $\mathbb{D}^{-}$such that the space $\mathcal{B}$ is an analytic subspace of $L^{t}(\mu)$. Suppose that $\mu(\mathbb{T})>0$. Then $\mathcal{M} \in \operatorname{Lat}(L, \mathcal{B}), \mathcal{M} \neq(0)$, contains all of the polynomials if and only if $M_{z} \mathcal{M} \subset \mathcal{M}$.

Proof. We will first show the sufficiency. Thus, assume that $\mathcal{M} \neq(0)$ is both $L$-invariant and $M_{z}$-invariant. A power series argument shows there is a $f \in \mathcal{M}$ with $f(0) \neq 0$. Then for all $n \in \mathbb{N} \cup\{0\}$

$$
-f(0) z^{n}=z^{n+1} L f-z^{n} f \in \mathcal{M} .
$$

Thus $\mathcal{M}$ contains all of the polynomials.

We will prove the converse by contradiction. Suppose that $\mathcal{M}$ is not $M_{z^{-}}$ invariant but that $\mathcal{M}$ contains all of the polynomials. Then there is an $f_{0} \in \mathcal{M}$ 
such that $\left[f_{0}\right] \not \subset \mathcal{M}$ and hence there is a $h \in \mathcal{M}^{\perp}$ such that $C_{\mu}\left(f_{0} \bar{h}\right) \not \equiv 0$ on $\mathbb{D}_{e}$. Since $h \in \mathcal{M}^{\perp}$ and $\mathcal{M}$ contains all of the polynomials,

$$
\int \frac{\bar{h}}{z-\lambda} d \mu=0, \quad \forall \lambda \in \mathbb{D}_{e}
$$

and thus if we apply Lemma 4.5 with $f=1$ we obtain that

$$
\left(\frac{1}{f_{0}}\right)_{r} \rightarrow 0 \text { in measure }[|d \zeta|] \text { on } \mathbb{T} \text { as } r \rightarrow 1^{-} \text {. }
$$

However, since $f_{0} \in L^{t}(\mu)$ we have that $\mid f_{0} \| \mathbb{T}<+\infty[\mu]$-a.e. and thus (4.12) contradicts the hypothesis together with part 2 of Proposition 4.2

As mentioned in Section 1, for special measures, we can improve Theorem 4.6.

Theorem 4.8. Let $\mu$ be of the form (1.9) and $1 \leq t<+\infty$. If $\mathcal{M} \in$ $\operatorname{Lat}\left(L, L_{a}^{t}(\mu)\right)$ does not contain all of the polynomials, then $\mathcal{N} \subset \mathfrak{N}(\mathbb{D})$ and every $f \in \mathcal{M}$ has a pseudocontinuation across $\mathbb{T}$ to a function in $\mathfrak{N}\left(\mathbb{D}_{e}\right)$.

Proof. By Theorem 4.6 and Fatou's theorem, it suffices to show that $\mathcal{M} \subset$ $\mathfrak{N}(\mathbb{D})$.

Let $f \in \mathcal{M}$. For $g \in \mathcal{M}^{\perp}$, we follow exactly as in the proof of Theorem 4.6 to conclude that for each

$$
r \in E=E_{g d \mu} \cap E_{f g d \mu}
$$

$$
f(r \zeta) \int \frac{\bar{g}}{z-r \zeta} d \mu=\int \frac{f \bar{g}}{z-r \zeta} d \mu \quad[|d \zeta|] \text {-a.e. }|\zeta|=1 .
$$

For $r \in E$, and $|\lambda|<1$ define

$$
G_{r}(\lambda)=\int_{|z|<r} \frac{g(z)}{\bar{z}-r / \lambda} d \mu(z)
$$

and note that from Lemma $3.2, G_{r} \in H^{p}(\mathbb{D})$ for all $0<p<1$ and

$$
\left\|G_{r}\right\|_{H^{p}} \leq c_{p} \int|g| d \mu \quad \forall r \in(0,1) \cap E .
$$

Moreover $($ since $\mu(\mathbb{T})=0)$

$$
G_{r}(\lambda) \rightarrow G(\lambda) \equiv \int_{|z|<1} \frac{g}{\bar{z}-\frac{1}{\lambda}} d \mu
$$


uniformly on compact sets as $r \rightarrow 1^{-}, r \in E$. By Fatou's lemma and (4.14),

$$
\|G\|_{H^{p}} \leq \sup _{r \in(0,1) \cap E}\left\|G_{r}\right\|_{H^{p}}<+\infty
$$

and so $G \in H^{p}(\mathbb{D})$.

A power series computation yields

$$
G(\lambda)=-\lambda \sum_{n=0}^{\infty} \lambda^{n} \int_{|z|<1} \bar{z}^{n} g d \mu .
$$

But recall that since $\mathcal{M}$ does not contain the polynomials, $g$ can be chosen so that it does not annihilate all the polynomials and so $G \not \equiv 0$.

Since $r \in E$ we can apply a similar argument as was used to prove (3.6) to get that $G_{r}(\zeta)$, the non-tangential boundary values of the $H^{p}$ function $G_{r}$, are given $[|d \zeta|]$-a.e. by

$$
G_{r}(\zeta)=\int_{|z|<r} \frac{g(z)}{\bar{z}-r \bar{\zeta}} d \mu(z) .
$$

Let $\psi$ be the bounded analytic function in the definition of $\mu$ as in (1.9). Our first step is to show that for some $0<\delta<\gamma$

$$
\int_{|\zeta|=1}|\psi(r \zeta) f(r \zeta)|^{\delta}\left|G_{r}(\zeta)\right|^{\gamma}|d \zeta|
$$

remains uniformly bounded for $r \in E$.

To this end, we let $\varepsilon>0$ be as in (1.9) and choose $0<\delta<1 / 4$ so that $\delta / \varepsilon<1 / 4$. Letting $\gamma=\delta(1+\varepsilon) / \varepsilon$ we have

$$
\begin{aligned}
\gamma-\delta & <\frac{1}{4} \\
\gamma & <\frac{1}{2} .
\end{aligned}
$$

Now note that

$$
\left|G_{r}(\zeta)\right|=\left|\overline{G_{r}(\zeta)}\right|=\left|\int_{|z|<r} \frac{\bar{g}}{z-r \zeta} d \mu\right|
$$

and so

$$
\begin{aligned}
& \quad|\psi(r \zeta) f(r \zeta)|^{\delta}\left|G_{r}(\zeta)\right|^{\gamma} \\
& \leq|\psi(r \zeta) f(r \zeta)|^{\delta}\left|\int_{|z|>r} \frac{\bar{g}}{z-r \zeta} d \mu\right|^{\gamma} \\
& \quad+|\psi(r \zeta)|^{\delta}\left|f(r \zeta) \int_{|z|<1} \frac{\bar{g}}{z-r \zeta} d \mu\right|^{\delta}\left|\int_{|z|<1} \frac{\bar{g}}{z-r \zeta} d \mu\right|^{\gamma-\delta} .
\end{aligned}
$$


By (4.13), the second term above is equal to

$$
|\psi(r \zeta)|^{\delta}\left|\int_{|z|<1} \frac{f \bar{g}}{z-r \zeta} d \mu\right|^{\delta}\left|\int_{|z|<1} \frac{\bar{g}}{z-r \zeta} d \mu\right|^{\gamma-\delta}
$$

Since $\delta<1 / 4$ and $\gamma-\delta<1 / 4$ (see (4.16) and (4.17)), we can use the CauchySchwarz inequality along with Lemma 3.2 to get that the integral $|d \zeta|$ of the second term in the estimate of (4.18) is $O(1)$.

We now estimate the first term in the estimate of (4.18). At this point, we should mention that in some cases, i.e., when $\mu$ is a radial measure and $g$ is analytic, then this term is zero. In the general case, we have by the CauchySchwarz inequality, the $|d \zeta|$ integral of this term is bounded by

$$
C\left(\int_{|\zeta|=1}|\psi(r \zeta) f(r \zeta)|^{2 \delta}|d \zeta|\right)^{1 / 2}\left(\int_{|\zeta|=1}\left|\int_{|z|>r} \frac{\bar{g}}{z-r \zeta} d \mu\right|^{2 \gamma}|d \zeta|\right)^{1 / 2}
$$

By Lemma 3.2 (note $2 \gamma<1$ by (4.17)) this is bounded by

$$
C\left(\int_{|\zeta|=1}|\psi(r \zeta) f(r \zeta)|^{2 \delta}|d \zeta|\right)^{1 / 2}\left(\int_{|z|>r}|g| d \mu\right)^{\gamma}
$$

Now note that $|\psi f|^{2 \delta}$ is subharmonic on $\mathbb{D}$ and so the integrals

$$
\int_{|\zeta|=1}|\psi(r \zeta) f(r \zeta)|^{2 \delta}|d \zeta|
$$

increase as $r \rightarrow 1^{-}$and so (4.19) is bounded by

$$
\left(\frac{1}{\nu([r, 1))} \int_{|z|>r}|\psi f|^{2 \delta}|d \zeta| d \nu\right)^{1 / 2}\left(\int_{|z|>r}|g| d \mu\right)^{\gamma}
$$


Using Hölder's inequality with $p=t / 2 \delta>1$ (since $\delta<1 / 4)$ we get

$$
\begin{aligned}
& \left(\int_{|z|>r}|\psi f|^{2 \delta}|d \zeta| d \nu\right)^{1 / 2} \\
= & \left(\int_{|z|>r}|f|^{2 \delta}|\psi|^{2 \delta / t}|\psi|^{2 \delta-2 \delta / t}|d \zeta| d \nu\right)^{1 / 2} \\
\leq & \left(\int_{|z|>r}|f|^{t}|\psi||d \zeta| d \nu\right)^{\delta / t}\left(\int_{|z|>r}|\psi|^{(2 \delta-2 \delta / t)(t /(t-2 \delta))}|d \zeta| d \nu\right)^{1 / 2-\delta / t} \\
\leq & C\left(\int_{|z|>r}|f|^{t} d \mu\right)^{\delta / t}\left(\nu([r, 1))^{1 / 2-\delta / t} .\right.
\end{aligned}
$$

Notice in the last inequality, we use the fact that $|\psi| \leq w$.

Also by Hölder's inequality,

$$
\left(\int_{|z|>r}|g| d \mu\right)^{\gamma} \leq\left(\int_{|z|>r}|g|^{s} d \mu\right)^{\gamma / s}\left(\mu(\{|z|>r\})^{\gamma / t} .\right.
$$

Recall that $s$ is the conjugate index to $t^{12}$. From this we see that (4.20) is

$$
O\left(\mu(\{|z|>r\})^{\gamma / t} \nu([r, 1))^{-\delta / t}\right)
$$

and since $w \in L^{1+\varepsilon}(d \nu|d \zeta|)$ we have

$$
\mu(\{|z|>r\}) \leq\left(\int_{|z|>r} w^{1+\varepsilon} d \nu|d \zeta|\right)^{1 /(1+\varepsilon)}\left(\int_{|z|>r} d \nu|d \zeta|\right)^{\varepsilon /(1+\varepsilon)} .
$$

Thus

$$
\mu(\{|z|>r\})^{\gamma / t}=O\left(\nu([r, 1))^{\varepsilon \gamma / t(1+\varepsilon)}\right)
$$

and so by combining this with (4.21) we see that (4.19) is

$$
O\left(\nu([r, 1))^{\varepsilon \gamma / t(1+\varepsilon)-\delta / t}\right)
$$

which is $O(1)$ since $\gamma=(1+\varepsilon) \delta / \varepsilon$.

Thus we have shown that (4.15) is uniformly bounded for $r \in E$. Now we will show that $f$ is a Nevanlinna function on the disk. Let $O_{G_{r}}$ be the outer factor of $G_{r}$ and notice from (4.14)

$$
\int_{|\zeta|=1}\left|O_{G_{r}}^{\gamma / \delta}\right|^{\delta}|d \zeta| \leq \int_{|\zeta|=1}\left|G_{r}\right|^{\gamma}|d \zeta| \leq c_{\gamma}\left(\int|g| d \mu\right)^{\gamma}
$$

12 Here one needs to make the obvious modification if $t=1$ and $s=\infty$. 
and hence $O_{G_{r}}^{\gamma / \delta}$ is uniformly bounded on compact subsets of $\mathbb{D},[21]$, p. 36. By a normal families argument

$$
O_{G_{r_{n}}}^{\gamma / \delta}(\lambda) \rightarrow H(\lambda)
$$

uniformly on compact sets for some sequence $r_{n} \rightarrow 1$ with $r_{n} \in E$. By Fatou's lemma $H \in H^{\delta}(\mathbb{D})$. Also note that since $G \not \equiv 0$, then $H \not \equiv 0$. In a similar way, since

$$
\int_{|\zeta|=1}\left|\psi(r \zeta) f(r \zeta) O_{G_{r}}^{\gamma / \delta}(\zeta)\right|^{\delta}|d \zeta|
$$

is uniformly bounded for $r \in E$, then by normal families

$$
\psi\left(r_{n} \lambda\right) f\left(r_{n} \lambda\right) O_{G_{r_{n}}}^{\gamma / \delta}(\lambda) \rightarrow K(\lambda)
$$

uniformly on compact subsets of $\mathbb{D}$ for some $r_{n} \rightarrow 1^{-}$. By Fatou's lemma, $K \in H^{\delta}(\mathbb{D})$ and so $\psi f H=K$ with $H \not \equiv 0$ and so $f=K / \psi H$ is a Nevanlinna function.

\section{Duality}

As mentioned in Section 1, for many Banach spaces of analytic functions $\mathcal{B}$ satisfying (1.1) through (1.5), there is a natural correspondence between $\operatorname{Lat}(L, \mathcal{B})$ and $\operatorname{Lat}\left(M_{z}, \mathcal{D}\right)$, where $\mathcal{D}$ is a certain dual space via the " $H^{2}$-duality". This correspondence will be important to us for two reasons. First, we will let $\mathcal{B}=P^{t}(\mu)$ and use this duality along with the spectral results in Section 4 to show that for a large class of weighted Dirichlet spaces and Besov spaces (defined below) every non-zero $M_{z}$-invariant subspace of $\mathcal{D}$ has index equal to one. Secondly, we will use this duality in the next section to begin to examine the $L$-invariant subspaces of the Besov classes where the spectral situation is quite different from that of the analytic subspaces of $L^{t}(\mu)$. The duality mentioned above is well known but we review it here for the sake of completeness.

Let $\mathfrak{B}$ be a Banach space of analytic functions on $\mathbb{D}$ which satisfy conditions (1.1) through (1.5) with the two additional conditions

$$
\text { the polynomials are dense in } \mathfrak{B} \text {, }
$$

Conditions (1.3) and (1.5) for $\mathfrak{B}$ say that

$$
\frac{1}{1-\bar{\lambda} z} \in \mathfrak{B} \quad \forall \lambda \in \mathbb{D}
$$


and so for each $\phi \in \mathfrak{B}^{*}$ we can define the function

$$
(U \phi)(\lambda)=f(\lambda)=\left\langle\phi, \frac{1}{1-\bar{\lambda} z}\right\rangle_{\left(\mathfrak{B}^{*}, \mathfrak{B}\right)} .
$$

Notice that $U \phi \equiv 0$ if and only if $\phi$ annihilates $1 /(1-\bar{\lambda} z)$ for each $\lambda \in \mathbb{D}$, which by Proposition 2.2 and hypothesis (5.1) implies that $\phi=0$. Thus $U$ is injective and so we may define the space

$$
\mathfrak{D}=U \mathfrak{B}^{*}
$$

with norm given by

$$
\|f\|_{\mathfrak{D}}=\|\phi\|_{\mathfrak{B}^{*}} .
$$

Remark 5.1. For technical purposes we will assume that all our dual pairings $\langle\cdot, \cdot\rangle$ are linear in the first slot and conjugate linear in the second slot. Thus the function $(U \phi)(\lambda)$ defined above is analytic on $\mathbb{D}$.

\section{Proposition 5.2.}

(1) $\mathfrak{D}$ is a Banach space of analytic functions on $\mathbb{D}$ which satisfies properties (1.1) through (1.5).

(2) The polynomials are dense in $\mathfrak{D}$.

(3) The map $U^{*}: \mathfrak{D}^{*} \rightarrow \mathfrak{B}$ is onto and given by the formula

$$
\left(U^{*} F\right)(z)=\left\langle F, \frac{1}{1-\lambda \bar{z}}\right\rangle_{\left(\mathfrak{D}^{*}, \mathfrak{D}\right)} \quad \text { see footnote } 13 .
$$

(4) $(L \mid \mathfrak{B}) U^{*}=U^{*}\left(M_{z} \mid \mathfrak{D}\right)^{*}$

(5) $(L \mid \mathfrak{D}) U=U\left(M_{z} \mid \mathfrak{B}\right)^{*}$

Proof. Property (1.1) for $\mathfrak{D}$ follows from the definition and from what was said above.

Since $\sigma\left(\left(M_{z} \mid \mathfrak{B}\right)\right)=\mathbb{D}^{-}$(property $(1.5)$ for $\mathfrak{B}$ ), then for all $w \in \mathbb{D}$ the operator $\left(M_{z} \mid \mathfrak{B}\right)^{*}\left(I-\bar{w}\left(M_{z} \mid \mathfrak{B}\right)^{*}\right)^{-1}$ is continuous on $\mathfrak{B}^{*}$. Let

$$
L_{w, \mathfrak{D}} f=\frac{f-f(w)}{z-w}
$$

and note that a short computation shows that for $\phi \in \mathfrak{B}^{*}$

$$
\left(L_{w, \mathfrak{D}} U \phi\right)(\lambda)=\left(U\left(M_{z} \mid \mathfrak{B}\right)^{*}\left(I-\bar{w}\left(M_{z} \mid \mathfrak{B}\right)^{*}\right)^{-1} \phi\right)(\lambda) .
$$

Thus $L_{w, \mathfrak{D}}$ is continuous on $\mathfrak{D}$ which proves condition (1.4) for $\mathfrak{D}$ as well as (5). In a very similar way, one proves (4) which proves condition (1.2) for $\mathfrak{D}$.

${ }^{13}$ Here we are using the fact that $\mathfrak{B}$ is reflexive. 
In Section 2 we showed that $\sigma(L \mid \mathfrak{B})=\mathbb{D}^{-}$and so by using (4), one can show property (1.4) for $\mathfrak{D}$.

By property (1.1) for $\mathfrak{B}$, the linear functional $g \rightarrow g(z)$ is continuous on $\mathfrak{B}$ for all $z \in \mathbb{D}$ and thus for each $z \in \mathbb{D}$, there is a $\phi_{z} \in \mathfrak{B}^{*}$ with

$$
\left(U \phi_{z}\right)(\lambda)=\left\langle\phi_{z}, \frac{1}{1-\bar{\lambda} w}\right\rangle_{\left(\mathfrak{B}^{*}, \mathfrak{B}\right)}=\frac{1}{1-\lambda \bar{z}} .
$$

Thus $\left\{(1-\lambda \bar{z})^{-1}: z \in \mathbb{D}\right\} \subset \mathfrak{D}$ and so property (1.3) holds for $\mathfrak{D}$.

For $F \in \mathfrak{D}^{*}$

$$
\begin{aligned}
\left\langle F, \frac{1}{1-\lambda \bar{z}}\right\rangle_{\left(\mathfrak{D}^{*}, \mathfrak{D}\right)} & =\left\langle F, U \phi_{z}\right\rangle_{\left(\mathfrak{D}^{*}, \mathfrak{D}\right)} \\
& =\left\langle U^{*} F, \phi_{z}\right\rangle_{\left(\mathfrak{B}, \mathfrak{B}^{*}\right)} \\
& =\left(U^{*} F\right)(z)
\end{aligned}
$$

This shows formula (3). To show that $U^{*}$ is onto $\mathfrak{B}$ we note that if $\phi \in \mathfrak{B}^{*}$ with $\left\langle\phi, U^{*} F>_{\left(\mathfrak{B}^{*}, \mathfrak{B}\right)}=0 \forall F \in \mathfrak{D}^{*}\right.$, then $\left\langle U \phi, F>_{\left(\mathfrak{D}, \mathfrak{D}^{*}\right)}=0 \forall F \in \mathfrak{D}^{*}\right.$ which means that $U \phi$ and hence $\phi$ are zero. By the Hahn-Banach theorem $U^{*}$ is onto.

Finally, to prove that polynomials are dense in $\mathfrak{D}$ we note that by property (1.1) for $\mathfrak{B}$, the span of $\left\{\phi_{z}: z \in \mathbb{D}\right\}$ equals $\mathfrak{B}^{*}$ and so $\left\{\left(U \phi_{z}\right)(\lambda)=(1-\lambda \bar{z})^{-1}\right.$ : $z \in \mathbb{D}\}$ spans $\mathfrak{D}$. By Proposition 2.2 this means that polynomials are dense in $\mathfrak{D}$.

\section{Remark 5.3.}

(1) Note that for polynomials $p \in \mathfrak{B}$ and $q \in \mathfrak{D}$ the dual pairing between $\mathfrak{B}$ and $\mathfrak{D}$ is given by

$$
\langle p, q\rangle_{(\mathfrak{B}, \mathfrak{D})}=\int p(\zeta) \overline{q(\zeta)} \frac{|d \zeta|}{2 \pi}
$$

which is why it is called the " $H^{2}$-duality".

(2) We point out that if in the above construction $\mathcal{B}^{*}$ is replaced with an isomorphic space $\mathcal{X}$, then the corresponding function space $\mathfrak{D}$ remains unchanged (with an equivalent norm).

Definition 5.4. We introduce the following classes of functions which will be used below as well as in the later sections. Let $\alpha>-1$ and $1<p<+\infty$.

(1) The weighted Bergman spaces

$$
A_{\alpha}^{p}=\left\{f \in \operatorname{Hol}(\mathbb{D}): f \in L^{p}\left((1-|z|)^{\alpha} d A\right)\right\} .
$$


(2) The Besov spaces

$$
X_{\alpha, p}=\left\{h \in \operatorname{Hol}(\mathbb{D}): h^{(n+1)}\left(1-|z|^{2}\right)^{n-\alpha} \in L^{p}\left(\left(1-|z|^{2}\right)^{\alpha} d A\right)\right\} \text { see footnote } 14
$$

(3) The unweighted Bergman spaces $L_{a}^{p}=A_{0}^{p}$.

(4) The $L^{p}$-Dirichlet spaces $\mathcal{D}_{p}=X_{0, p}=\left\{f \in \operatorname{Hol}(\mathbb{D}): f^{\prime} \in L_{a}^{p}\right\}$.

Let $1<p<+\infty, \alpha>-1$, and $1 / p+1 / q=1$.

Example 5.5. It is well known [31] that $\left(A_{\alpha}^{p}\right)^{*} \simeq X_{\alpha, q}$ via the pairing

$$
\langle f, g\rangle=\lim _{r \rightarrow 1^{-}} \int_{|\zeta|=1} f(r \zeta) \overline{g(r \zeta)} \frac{|d \zeta|}{2 \pi} .
$$

Thus in this case, if $\mathfrak{B}=A_{\alpha}^{p}$, then $\mathfrak{B}^{*}=X_{\alpha, q}$ and $U \phi=\phi$ and so $\mathfrak{D}=X_{\alpha, q}$. In a similar way, if $\mathfrak{B}=X_{\alpha, p}$, then $\mathfrak{D}=A_{\alpha}^{q}$.

Example 5.6. One has $[10]\left(L_{a}^{p}\right)^{*} \simeq L_{a}^{q}$ via the pairing

$$
\langle f, g\rangle=\int_{\mathbb{D}} f(z) \overline{g(z)} \frac{d A}{\pi}
$$

Thus if $\mathfrak{B}=L_{a}^{p}(d A)$, then $\mathfrak{B}^{*}=L_{a}^{q}(d A)$ and for $\phi \in L_{a}^{q}(d A)$, one computes

$$
(U \phi)(\lambda)=\frac{1}{\lambda} \int_{0}^{\lambda} \phi(z) d z
$$

and so $\mathfrak{D}=U L_{a}^{q}(d A)=\mathcal{D}_{q}$.

Example 5.7. Similarly, $\left(\mathcal{D}_{p}\right)^{*} \simeq \mathcal{D}_{q}{ }^{15}$ via the pairing

$$
\langle f, g\rangle=\int_{|\zeta|=1} f(\zeta) \overline{g(\zeta)} \frac{|d \zeta|}{2 \pi}+\int_{\mathbb{D}} f^{\prime}(z) \overline{g^{\prime}(z)} \frac{d A}{\pi}=\int_{\mathbb{D}}(z f)^{\prime}(z) \overline{(z g)^{\prime}(z)} \frac{d A}{\pi}
$$

Thus if $\mathfrak{B}=\mathcal{D}_{p}$. Then for $\phi \in \mathfrak{B}^{*}=\mathcal{D}_{q}$

$$
(U \phi)(\lambda)=(z \phi)^{\prime}(\lambda)
$$

and hence $\mathfrak{D}=U \mathcal{D}_{q}=L_{a}^{q}(d A)$.

${ }^{14}$ ( $n$ is a integer with $n \geq \alpha$ ). It is known [31] that that for $1<p<+\infty$ the definition of $X_{\alpha, p}$ is independent of the choice of $n \geq \alpha$. There is also a description of these functions in terms of their boundary values on $\mathbb{T}$.

15 This is just a special case of our first example but we point it out here since we will be using this particular pairing later on in the paper. 
Example 5.8. Let $1<t<+\infty$ and let $\mu$ be a measure on $\mathbb{D}^{-}$such that $P^{t}(\mu) \hookrightarrow \operatorname{Hol}(\mathbb{D})$. We define

$$
D(t, \mu)=U\left(P^{t}(\mu)\right)^{*}=\left\{F: F(\lambda)=\int_{\mathbb{D}^{-}} \phi(z) \frac{1}{1-\lambda \bar{z}} d \mu(z): \phi \in L^{s}(\mu)\right\}
$$

with norm

$$
\|F\|_{D(t, \mu)}=\|\phi\|_{L^{s} /\left(P^{t}\right)^{\perp}} .
$$

By our results on Cauchy transforms in Section 3, we observe that $D(t, \mu) \subset H^{p}$ for all $0<p<1$. If $\mu$ is a radial measure $d \mu=d \nu|d \zeta|$, and $t=2$, then this construction can be made very concrete. Indeed in this case, one checks that

$$
D(2, \mu)=\left\{\sum_{n} a_{n} z^{n}: \sum_{n}\left(\int_{0}^{1} r^{2 n} d \nu(r)\right)^{-1}\left|a_{n}\right|^{2}<+\infty .\right\} .
$$

As to be expected, in this case we even have $D(2, \mu) \subset H^{2}$ and $M_{z} \mid D(2, \mu)$ is a weighted forward shift operator.

If $\mathcal{N}$ is a subspace of $\mathfrak{D}$ and $\mathcal{M}=U^{*} \mathcal{N}^{\perp}$, then from Proposition 5.2 we see that

$$
\mathcal{N} \in \operatorname{Lat}\left(M_{z}, \mathfrak{D}\right) \Leftrightarrow \mathcal{M} \in \operatorname{Lat}(L, \mathfrak{B}) .
$$

Recall that $\mathcal{N} \in \operatorname{Lat}\left(M_{z}, \mathfrak{D}\right)$ has index one if $\operatorname{dim}(\mathcal{N} / z \mathcal{N})=1$. The index one property has also been called the "division property" or "codimension one property" and has been studied in [6] [35]. The analog of the next result, stated for $M_{z}^{*} \mid \mathfrak{D}^{*}$ instead of $L \mid \mathfrak{B}$, is from [35], Theorem 4.5. However, one can provide an alternative proof which uses Proposition 2.8. We leave this as an exercise to the reader.

Proposition 5.9. Suppose $\mathcal{N} \in \operatorname{Lat}\left(M_{z}, \mathfrak{D}\right), \mathcal{N} \neq(0)$, and $\mathcal{N}=U^{*} \mathcal{N}{ }^{\perp}$. If $Z(\mathcal{N})$ denotes the set of common zeros of the functions in $\mathcal{N}$ in $\mathbb{D}$, we conclude the following:

(1) If $\operatorname{ind}(\mathcal{N})=1$, then $\sigma(L \mid \mathcal{M}) \cap \mathbb{D}=\{\bar{\lambda}: \lambda \in Z(\mathcal{N})\}$.

(2) If $\operatorname{ind}(\mathcal{N})>1$, then $\sigma(L \mid \mathcal{M})=\mathbb{D}^{-}$.

If $\mathfrak{B}=P^{t}(\mu)$, then $\mathfrak{D}=D(t, \mu)$. Also, if $\mathfrak{B}=A_{\alpha}^{q}$, then $\mathfrak{D}=X_{\alpha, p}$ and thus applying Theorem 4.6 we have the following corollary:

Corollary 5.10. Let $1<p, t<+\infty$ and $\alpha>-1$. If $\mathfrak{D}=D(t, \mu)$ or $\mathfrak{D}=X_{\alpha, p}$ and $\mathcal{N} \in \operatorname{Lat}\left(M_{z}, \mathfrak{D}\right), \mathcal{N} \neq(0)$, then $\operatorname{ind}(\mathcal{N})=1$.

We mention that this was shown for $D(2, d A)$ in [37] and for $D\left(2,(1-|z|)^{\alpha} d A\right)$ in [5]. In the case where $D(t, \mu)$ is a Banach algebra this was observed in [13]. 


\section{Remark 5.11.}

(1) From Corollary 5.10 we see that given a sequence $\left\{a_{n}\right\} \subset \mathbb{D}$, there is a $\mathcal{M} \in \operatorname{Lat}(L, \mathfrak{B})$ with $\sigma(L \mid \mathcal{M}) \cap \mathbb{D}=\left\{a_{n}\right\}$ if and only if there is a $f \in \mathfrak{D}$ such that $f^{-1}(\{0\})=\left\{a_{n}\right\}$.

(2) The collection $\{\sigma(L \mid \mathcal{M}) \cap \mathbb{D}: \mathcal{M} \in \operatorname{Lat}(L, \mathfrak{B})\}$ can be quite different for various spaces $\mathfrak{B}$. For example if $\mathfrak{B}=L_{a}^{t}(d A)$, then $\mathfrak{D}=\mathcal{D}_{s}(1 / s+1 / t=$ 1 ) and so for any $\mathcal{M} \in \operatorname{Lat}\left(L, L_{a}^{t}(d A)\right), \mathcal{M} \neq L_{a}^{t}(d A)$, the spectrum in the disk must be a Blaschke sequence. However for $s>2, \mathcal{D}_{s}$ is contained in some Lipschitz class and thus the zeros of a (non-zero) function must satisfy the condition

$$
\int_{|\zeta|=1} \log \operatorname{dist}\left(\zeta,\left\{a_{n}\right\}\right)|d \zeta|>-\infty
$$

and in particular cannot accumulate on any set of positive measure [44]. On the other hand, if $t=2$, then the zeros of a $\mathcal{D}_{2}$ function can accumulate on all of $\mathbb{T}[15]$. As another example, if $\mathfrak{B}=\mathcal{D}_{t}$, then $\mathfrak{D}=L_{a}^{s}(d A)$ and it is well known that the zeros of Bergman space functions can be quite complicated and need not be Blaschke [26] [27] [28]. Thus in contrast to the Bergman space, even though $\sigma_{\text {ap }}(L \mid \mathcal{M}) \cap \mathbb{D}$ is discrete, it need not be Blaschke.

\section{The BaCKWARD ShIFT ON The Besov Classes}

Motivated by the discussion in the previous section, we proceed to investigate the properties of the restriction of $L$ to its invariant subspaces of the Besov classes $X_{\alpha, p}$. As is to expect, the spectral and pseudocontinuation situation is strikingly different to the one in the weighted Hardy and Bergman spaces. Recall that for these spaces, we saw that $\sigma(L \mid \mathcal{M})=\sigma_{\mathrm{ap}}(L \mid \mathcal{M})$. Let us first record the following "negative" result concerning the spectrum of an $L$-invariant subspace.

Proposition 6.1. Given $\alpha>-1$ and $1<p<+\infty$, there is an $\mathcal{M} \in$ $\operatorname{Lat}\left(L, X_{\alpha, p}\right)$ such that $\sigma(L \mid \mathcal{M})=\mathbb{D}^{-}, \sigma_{\mathrm{ap}}(L \mid \mathcal{M})=\mathbb{T}$, and $\sigma_{p}(L \mid \mathcal{M})=\emptyset$.

Proof. Recall from Section 5 the duality between $A_{\alpha}^{q}$ and $X_{\alpha, p}$ and so for any $\mathcal{N} \in \operatorname{Lat}\left(M_{z}, A_{\alpha}^{q}\right)$ with ind $(\mathcal{N})>1$ we have from Proposition 5.9

$$
\mathbb{D}^{-}=\sigma\left(L \mid \mathcal{N}^{\perp}\right) \supset \sigma_{\text {ap }}\left(L \mid \mathcal{N}^{\perp}\right) \supset \partial \sigma\left(L \mid \mathcal{N}^{\perp}\right)=\mathbb{T} .
$$

The existence of such invariant subspaces has been proved in [11] [22] [27]. Moreover, by Proposition 2.1 and the identity

$$
\left\langle f, \frac{1}{1-\bar{a} z}\right\rangle=f(a), \quad \forall f \in A_{\alpha}^{q}, a \in \mathbb{D},
$$


the equality $\sigma_{\text {ap }}\left(L \mid \mathcal{N}^{\perp}\right)=\mathbb{T}$ is equivalent to the fact that $\mathcal{N}$ has no common zeros. An explicit construction of such invariant subspaces can be found in [26] and [27].

Finally, from Proposition $2.1 \sigma_{p}(L \mid \mathcal{M}) \cap \mathbb{D}=\emptyset$ and so if $\zeta \in \sigma_{p}(L \mid \mathcal{M}) \cap \mathbb{T}$, then the function $(1-\zeta z)^{-1}$ would be the corresponding eigenvector. However, this function does not belong to $H^{1}$ which is a contradiction to the fact that $X_{\alpha, p} \subset H^{1}$.

It is a little more delicate problem to illustrate the lack of pseudocontinuations for functions in non-trivial $L$-invariant subspaces of $X_{\alpha, p}$. Recall from Section 4 that for a large class of weighted Bergman spaces, non-cyclic functions for $L$ have pseudocontinuations to $\mathfrak{N}\left(\mathbb{D}_{e}\right)$. This next result shows that this fact no longer hold in the context of Besov spaces.

Theorem 6.2. Given $\alpha>-1$ and $1<p<+\infty$ there is a function $f \in$ $X_{\alpha, p}$ such that $[f]_{L} \neq X_{\alpha, p}$ and $f$ does not have a pseudocontinuation across any set of positive measure in $\mathbb{T}$.

The function $f$ in the statement of the above theorem will be an element of the annihilator of a $M_{z}$-invariant subspace of $A_{\alpha}^{q}$ determined by a zero set. Using either the Ph.D. thesis of S. Walsh [47] or more recent density theorems of K. Seip [41], one can show that given $\alpha>-1$ and $1<p<+\infty$, there is a sequence $A \subset \mathbb{D}$ with

$$
\mathcal{J}(A) \equiv\left\{f \in A_{\alpha}^{q}:\left.f\right|_{A}=0\right\} \neq(0)
$$

and such that given any point $\zeta \in \mathbb{T}$, there exists a subsequence of $A$ which converges to $\zeta$ non-tangentially ${ }^{16}$.

Using the identity

$$
\left\langle f, \frac{1}{1-\bar{a} z}\right\rangle=f(a), \quad \forall f \in A_{\alpha}^{q}, a \in \mathbb{D},
$$

one shows

$$
\mathcal{M}(A)=\mathcal{J}(A)^{\perp}=\operatorname{span}_{X_{\alpha, p}}\left\{\frac{1}{1-\bar{a} z}: a \in A\right\} .
$$

Thus if the sequence $A$ is chosen so that $\mathcal{J}(A) \neq 0$, then by the results in Section $5, \mathcal{M}(A) \in \operatorname{Lat}\left(L, X_{\alpha, p}\right)$ and $\mathcal{M}(A) \neq X_{\alpha, p}$. We will show that we can choose non-zero constants $c_{n}$ so that the function

$$
f(z)=\sum_{n=1}^{\infty} \frac{c_{n}}{1-\overline{a_{n}} z}
$$

16 Sequences which accumulate non-tangentially at $[|d \zeta|]$-a.e. point in $\mathbb{T}$ are often called dominating sequences for $H^{\infty}[14]$. 
belongs to $X_{\alpha, p}$ (hence also to $\mathcal{M}(A)$ ) and has no pseudocontinuation across any subset of $\mathbb{T}$ of positive measure. This will be done by showing that if $f$ has a pseudocontinuation across $E$ to a function $G \in \mathfrak{M}\left(\mathbb{D}_{e}\right)$, then in fact $G=f$ (as defined by the series above) on $\mathbb{D}_{e}$. But since the poles of $f$ (i.e., $1 / \overline{a_{n}}$ ) and hence $G$ accumulate non-tangentially to every boundary point, then $G$ cannot possibly have non-tangential limits $[|d \zeta|]$-a.e. on $E$. We should also point out that by Proposition 5.9

$$
\sigma(L \mid \mathcal{M}(A)) \cap \mathbb{D}=\{\bar{a}: a \in A\}
$$

is discrete, but this subspace contains functions that have no pseudocontinuation across any part of $\mathbb{T}$.

We now begin our construction. For any Dirichlet region $\Omega \subset \mathbb{C}$ and $z \in \Omega$, let $\omega(z, \cdot, \Omega)$ denote the harmonic measure on $\partial \Omega$ evaluated at $z$. For a point $b \in \mathbb{D}_{e}$ and $0<r<1$ let

$$
H(b, r)=\left\{z \in \mathbb{D}_{e}:\left|\frac{z-b}{1-\bar{b} z}\right|<r\right\}
$$

denote the pseudo-hyperbolic ball about the point $b$. One can argue (using the fact that $\omega(z, E, \Omega)$ is the unique harmonic function which is 1 on $E$ and 0 on $\partial \Omega \backslash E)$ that

$$
\omega\left(z, \partial H(b, r), \mathbb{D}_{e} \backslash H(b, r)^{-}\right)=\frac{\log \left|\frac{1-\bar{b} z}{z-b}\right|}{\log 1 / r} .
$$

Now let $b_{n}=1 / \overline{a_{n}}$ (tacitly assuming that $a_{n} \neq 0$ ) and choose $\rho_{n}>0$ so that

$$
\begin{aligned}
& H\left(b_{n}, \rho_{n}\right) \cap H\left(b_{m}, \rho_{m}\right)=\emptyset, \quad \forall n \neq m . \\
& H\left(b_{n}, \rho_{n}\right) \subset\left\{\left|z-b_{n}\right|<\frac{1}{3}\left(\left|b_{n}\right|-1\right)\right\} .
\end{aligned}
$$

Now choose $r_{n}<\rho_{n}$ so that

$$
\sum_{n} \frac{\log 1 / \rho_{n}}{\log 1 / r_{n}}<\infty
$$

For a closed set $E \subset \mathbb{T}$ of positive measure, we write $\mathbb{T} \backslash E$ as the disjoint union of open $\operatorname{arcs} J_{n}=\left(e^{i \alpha_{n}}, e^{i \beta_{n}}\right)$. Letting $e^{i \theta_{n}}$ denote the midpoint of $J_{n}$, we form the "triangular" shaped region $T_{n}$ with vertices $e^{i \alpha_{n}},\left(1+\frac{1}{2}\left|J_{n}\right|\right) e^{i \theta_{n}}, e^{i \beta_{n}}$. 


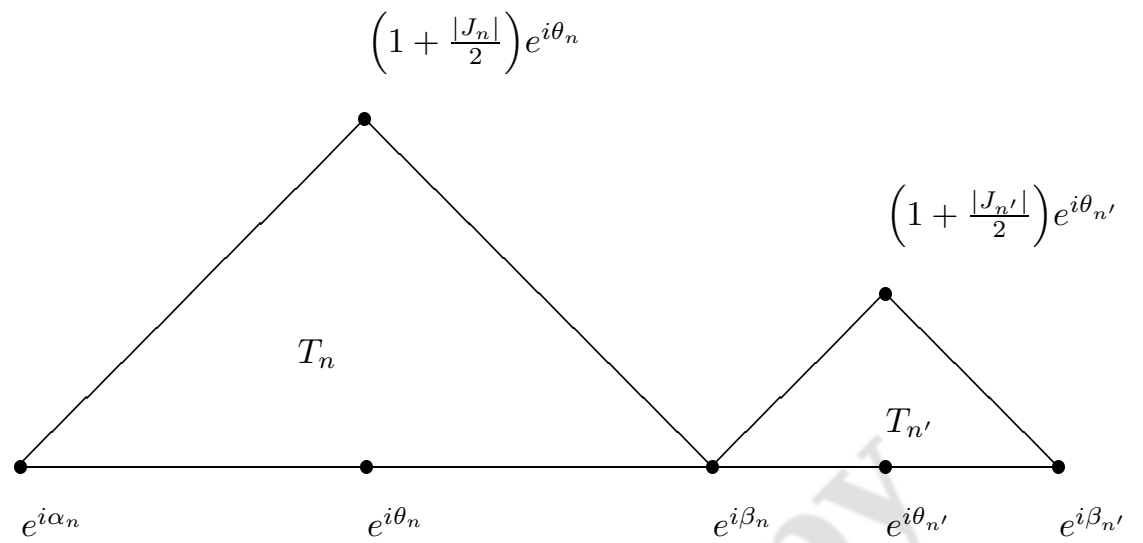

Notice that for any two $T_{n}$ and $T_{n^{\prime}}$ which share a common vertex (on $\mathbb{T}$ ), the angle formed at that vertex is $\pi / 2$. Moreover, one argues that the set

$$
\Omega(E)=\{1<|z|<3\} \backslash \bigcup_{n} T_{n}^{-}
$$

is an open connected subset of $\mathbb{D}_{e}$ whose boundary is a rectifiable curve whose length is comparable to

$$
6 \pi+\sum_{n} 2 \sqrt{2}\left|J_{n}\right|=6 \pi+\sqrt{2}|\mathbb{T} \backslash E|<+\infty .
$$

Moreover we also assume (by possibly adding a finite number of points on the circle to $E$ ) that

$$
\Omega(E)^{-} \cap \mathbb{T}=E
$$

(this only changes $E$ by a set of zero measure). Finally note that no component of $\mathbb{C}_{\infty} \backslash \Omega(E)$ reduces to a point and so $\Omega(E)$ is a Dirichlet region.

One argues using (6.3) the domain $\Omega(E)$ does not become disconnected when we remove any of the $H\left(b_{n}, r_{n}\right)$ and hence we can define the region

$$
R(E)=\Omega(E) \backslash \bigcup_{n} H\left(b_{n}, r_{n}\right)^{-} .
$$

Since no component of $\mathbb{C}_{\infty} \backslash R(E)$ reduces to a point, then $R(E)$ is a Dirichlet region.

Lemma 6.3. $\omega(z, E, R(E))>0$ for all $z \in R(E)$.

Proof. First we note that $\Omega(E)$ is a doubly connected region with rectifiable boundary and hence (for example see [17], p. 302)

$$
\omega(z, E, \Omega(E))>0, \quad z \in \Omega(E) .
$$


Next we notice that $z \rightarrow \omega(z, E, \Omega(E))$ is a bounded harmonic function on $\Omega(E)$ with uniquely determined boundary values (a.e. with respect to harmonic measure) ${ }^{17}$ which are 1 on $E$ and zero on $(\partial \Omega(E)) \backslash E$ and so

$$
\omega(z, E, \Omega(E))=\int \omega(\cdot, E, \Omega(E)) d \omega(z, R(E)), \quad z \in R(E)
$$

which is bounded above by

$$
\begin{aligned}
& \int_{E} d \omega(z, R(E))+\int_{\cup \partial H\left(b_{n}, r_{n}\right)} d \omega(z, R(E)) \\
& \quad \leq \omega(z, E, R(E))+\sum_{n} \omega\left(z, \partial H_{n}\left(b_{n}, r_{n}\right) \cap \partial R(E), R(E)\right) .
\end{aligned}
$$

For fixed $n$

$$
\omega\left(z, \partial H\left(b_{n}, r_{n}\right) \cap \partial R(E), R(E)\right) \leq \omega\left(z, \partial H\left(b_{n}, r_{n}\right), \mathbb{D}_{e} \backslash H\left(b_{n}, r_{n}\right)^{-}\right)
$$

which by (6.1) goes to zero as $|z| \rightarrow 1^{+}$. Furthermore for

$$
z \notin \bigcup_{n} H\left(b_{n}, \rho_{n}\right)
$$

we use (6.1) again to get

$$
\omega\left(z, \partial H\left(b_{n}, r_{n}\right) \cap \partial R(E), R(E)\right) \leq \frac{\log 1 / \rho_{n}}{\log 1 / r_{n}}
$$

which by (6.4) is summable in $n$. Thus by the dominated convergence theorem, the second term in (6.8) goes to zero as $|z| \rightarrow 1^{+}\left(z \notin H\left(b_{n}, \rho_{n}\right)\right.$ for any $\left.n\right)$. Combining this with (6.6) and (6.8) we see that $\omega(z, E, R(E))>0$ for $z$ near $\mathbb{T}$ and not in $H\left(b_{n}, \rho_{n}\right)$ for any $n$, and hence everywhere in $R(E)$.

Let $u: \mathbb{D} \rightarrow R(E)$ be an analytic covering map and notice that since $R(E)$ is a bounded region, then $u \in H^{\infty}(\mathbb{D})$ and so we can consider the set

$$
u^{-1}(E)=\{\zeta \in \mathbb{T}:|u(\zeta)|=1\}
$$

17 By boundary values, we mean the non-tangential limit values which are well defined a.e. due to the rectifiability of the boundary of $\Omega(E)$ 
(where $u(\zeta)$ denotes the non-tangential limit of $u$ at $\zeta$ ). Also notice from Lemma 6.3 that

$$
\begin{aligned}
\left|u^{-1}(E)\right| & =\lim _{n \rightarrow \infty} \int \frac{1}{|u(\zeta)|^{n}}|d \zeta|=\lim _{n \rightarrow \infty}\left\|\frac{1}{z^{n}}\right\|_{H^{1}(R(E))} \\
& =\int_{E} d \omega\left(z_{0}, R(E)\right) \\
& =\omega\left(z_{0}, E, R(E)\right)>0
\end{aligned}
$$

where $H^{1}(R(E))$ denotes the Hardy space on $R(E)$ with norming point $z_{0}=$ $u(0) \in R(E)$ [23], Chapter 2 .

Proof of Theorem 6.2. With the set up above, choose non-zero constants $\left\{c_{n}\right\}$ so that

$$
\begin{gathered}
f(z)=\sum_{n=1}^{\infty} \frac{c_{n}}{1-\overline{a_{n} z}} \in X_{\alpha, p} \\
f \text { is continuous on } \mathbb{C} \backslash \bigcup_{n} H\left(b_{n}, r_{n}\right) .
\end{gathered}
$$

Suppose that $f \mid \mathbb{D}$ has a pseudocontinuation $G \in \mathfrak{M}\left(\mathbb{D}_{e}\right)$ across some closed set $E \subset \mathbb{T}$ with $|E|>0$. By choosing a closed subset of positive measure, we can assume that the non-tangential limit of $G$ equals $f(\zeta)$ for each $\zeta \in E$. By construction, $f$ is continuous on $E$. Thus, by a standard argument (see [32], p. $83-84$ ), there will be a closed subset of $E$ of positive measure (also denoted by $E$ ) such that $G$ is continuous on $R(E)^{-}$except possibly for a finite number of poles. Thus, we can choose a polynomial $q$ such that $q G$ is continuous on $R(E)^{-}$.

Using the continuity of $f$ and $q G$ on $R(E)^{-}$we see that the function $(q f) \circ u$ and $(q G) \circ u$ are bounded analytic functions on $\mathbb{D}$ whose non-tangential limits agree $[|d \zeta|]$-a.e. on $u^{-1}(E)$ which has positive measure. Thus (since $u$ is onto) $f=G$ on $R(E)$ and hence $\mathbb{D}_{e}$. This means that $G$ has poles $\left\{1 / \overline{a_{n}}\right\}$ which by construction accumulate non-tangentially to every point on $\mathbb{T}$ and so $G$ cannot have a non-tangential limit $[|d \zeta|]$-a.e. on $E$, a contradiction. This completes the proof of the theorem.

\section{Remark 6.4.}

(1) We comment that Theorem 6.2 is true for a general Banach space of analytic functions $\mathcal{B}$ which satisfy conditions (1.1) through (1.5) along with the additional condition that

$$
\mathcal{B} \hookrightarrow X_{\alpha, p} \quad \text { for some } \alpha \text { and } p \text {. }
$$


One sees this by choosing (for the appropriate $\alpha$ and $p$ ) the sequence $A \subset \mathbb{D}$ as before and letting

$$
\mathcal{K}=\operatorname{span}_{\mathcal{B}}\left\{\frac{1}{1-\bar{a} z}: a \in A\right\} .
$$

From Proposition 2.2, $\mathcal{K}$ is contained in the closure of the polynomials in $\mathfrak{B}$ and so using this along with the continuous imbedding $\mathcal{B} \hookrightarrow X_{\alpha, p}$ and the density of polynomials in $X_{\alpha, p}$, we see that $\mathcal{K} \neq \mathcal{B}$. From our earlier construction, $\mathcal{K}$ has the right properties. Examples of such spaces include

$$
\begin{array}{ll}
A^{n}=\left\{f \in \operatorname{Hol}(\mathbb{D}): f^{(k)} \in C(\overline{\mathbb{D}}): 0 \leq k \leq n\right\}, & n \in \mathbb{N}, \\
H_{n}^{p}=\left\{f: f^{(k)} \in H^{p}: 0 \leq k \leq n\right\}, & n \in \mathbb{N}, 1 \leq p<+\infty .
\end{array}
$$

(2) One can choose the constants $c_{n}$ in the construction of the function $f$ above so that the $L$-invariant subspace generated by $f$ is in fact equal to $\mathcal{M}(A)$.

\section{The BaCKWard Shift on the Dirichlet SPaCES}

We have seen that not all $L$-invariant subspaces $\mathcal{M}$ of the Dirichlet spaces $\mathcal{D}_{p}, 1<p<+\infty$ satisfy that $\sigma(L \mid \mathcal{M}) \cap \mathbb{D}$ is discrete, because not all $M_{z^{-}}$invariant subspaces $\mathcal{N}$ of the Bergman spaces $L_{a}^{q}$ have index one. Since it is an important and unsolved question to determine which $M_{z}$ invariant subspaces of $L_{a}^{q}$ have index one, it becomes interesting to try to determine conditions on an $L$-invariant subspace $\mathcal{M}$ of $\mathcal{D}_{p}$ which will imply that $\sigma(L \mid \mathcal{M}) \cap \mathbb{D}$ is discrete. Despite the results of the previous section we will see that for certain $\mathcal{M} \in \operatorname{Lat}\left(L, \mathcal{D}_{p}\right)$ there is a connection between the existence of pseudocontinuations of the functions in $\mathcal{M}$ and the existence of $((1-\lambda L) \mid \mathcal{M})^{-1}$.

Fix $1<p<+\infty$ and let $\mathcal{N} \in \operatorname{Lat}\left(L, \mathcal{D}_{p}\right), \mathcal{M} \neq(0)$. The polynomials are dense in $\mathcal{D}_{p}$, hence it follows from Proposition 2.8 that $\sigma(L \mid \mathcal{M}) \cap \mathbb{D}$ is discrete if and only if for each $f \in \mathcal{M}$ the meromorphic function

$$
\lambda \rightarrow c_{\lambda}(f, \phi)=\left\langle\frac{z f}{z-\lambda}, \phi\right\rangle /\left\langle\frac{\lambda}{z-\lambda}, \phi\right\rangle, \quad \lambda \in \mathbb{D}_{e}
$$

is independent of $\phi \in \mathcal{M}^{\perp}, \phi \neq 0$. We shall show in this section that if for some $f \in \mathcal{M}$ and $\phi \in \mathcal{M}^{\perp}, c_{\lambda}(f, \phi)$ has a non-tangential limit on a set $E \subset \mathbb{T}$, then $c_{\lambda}(f, \phi)$ must be a pseudocontinuation of $f$ across $E$. Furthermore, we shall use this to obtain a condition on $\mathcal{M}^{\perp}$ which will imply that all functions in $\mathcal{M}$ have a pseudocontinuation across some $E \subset \mathbb{T}$ with $|E|>0$, and we shall use our results in turn to prove a theorem about index one $M_{z}$-invariant subspaces in the Bergman spaces. 
We start out by recalling some basic facts about $\mathcal{D}_{p}$. Recall that an analytic function $f$ on $\mathbb{D}$ belongs to the Dirichlet space $\mathcal{D}_{p}$ if it has finite $L^{p}$-Dirichlet integral

$$
D_{p}(f)=\frac{1}{\pi} \int_{\mathbb{D}}\left|f^{\prime}\right|^{p} d A .
$$

It is easy to show that $\mathcal{D}_{p} \subset H^{p}$ and so we can norm $\mathcal{D}_{p}$ by

$$
\|f\|_{\mathcal{D}_{p}}^{p}=\|f\|_{H^{p}}^{p}+D_{p}(f) .
$$

Moreover [43], Chapter 5, Section $5^{18}$, the above is equivalent to

$$
\|f\|_{H^{p}}^{p}+\int_{|w|=1} \int_{|z|=1}\left|\frac{f(z)-f(w)}{z-w}\right|^{p} \frac{|d z|}{2 \pi} \frac{|d w|}{2 \pi}
$$

and in fact, by a formula of J. Douglas [19], these two quantities are the same when $p=2$.

From Section 5 , the dual of $\mathcal{D}_{p}$ is $\mathcal{D}_{q}$ (where $q$ is the conjugate index to $p$ ) via the pairing

$$
\langle f, g\rangle=\int_{|\zeta|=1} f(\zeta) \overline{g(\zeta)} \frac{|d \zeta|}{2 \pi}+\frac{1}{\pi} \int_{\mathbb{D}} f^{\prime}(z) \overline{g^{\prime}(z)} d A(z) .
$$

For $\zeta \in \mathbb{T}$ and $f \in H^{p}$, it was shown that if

$$
\int_{|w|=1}\left|\frac{f(w)-\alpha}{w-\zeta}\right|^{p} \frac{|d w|}{2 \pi}<+\infty
$$

for some $\alpha \in \mathbb{C}$, then the non-tangential limit of $f$ at $\zeta$ equals $\alpha$ (see [39], p. 358$)^{19}$. Thus, as was done in [39] for $p=2$, we may define the $L^{p_{-} \text {"local }}$ Dirichlet integral" of $f$ at $\zeta \in \mathbb{T}$ to be

$$
D_{p, \zeta}(f)=\int_{|w|=1}\left|\frac{f(w)-f(\zeta)}{w-\zeta}\right|^{p} \frac{|d w|}{2 \pi} .
$$

For $f \in H^{p}$ and $g \in H^{q}$ and $\zeta \in \mathbb{T}$ for which $D_{p, \zeta}(f)$ and $D_{q, \zeta}(g)$ are finite, we can define, via Hölder's inequality, the mixed local Dirichlet integral as

$$
D_{\zeta}(f, g)=\int_{|w|=1} \frac{(f(w)-f(\zeta)) \overline{(g(w)-g(\zeta))}}{|w-\zeta|^{2}} \frac{|d w|}{2 \pi} .
$$
disk.

18 The proof there is for the upper-half plane but can easily be adapted to work for the 19 The proof cited is for $p=2$ but one can easily modify the proof to work for general $p$. 
Note that when $f \in \mathcal{D}_{p}, g \in \mathcal{D}_{q}$, then $D_{p, \zeta}(f)$ and $D_{\zeta}(f, g)$ are $L^{1}(\mathbb{T},|d \zeta|)$ functions. By the Douglas formula and the polarization identity, we note that for polynomials

$$
\langle f, g\rangle=\int_{|\zeta|=1} f(\zeta) \bar{g}(\zeta) \frac{|d \zeta|}{2 \pi}+\int_{|\zeta|=1} D_{\zeta}(f, g) \frac{|d \zeta|}{2 \pi} .
$$

But since polynomials are dense in $\mathcal{D}_{p}$, then this formula works for all $f \in \mathcal{D}_{p}$ and $g \in \mathcal{D}_{q}$.

Theorem 7.1. Let $1<p<+\infty, \mathcal{M} \in \operatorname{Lat}\left(L, \mathcal{D}_{p}\right), f \in \mathcal{M}, g \in \mathcal{M}^{\perp} \subset$ $\mathcal{D}_{q}, g \neq 0$. If there exists a set $E \subset \mathbb{T},|E|>0$, such that the meromorphic function $F$ on $\mathbb{D}_{e}, F(\lambda)=c_{\lambda}(f, g)$, has a non-tangential limit on $E$, then $F$ is a pseudocontinuation of $f$ across $E$.

Remark 7.2. $F$ may not be in the Nevanlinna class of $\mathbb{D}_{e}$.

In order to prove Theorem 7.1, we need two preliminary lemmas.

Lemma 7.3. Let $\mathcal{M} \in \operatorname{Lat}\left(L, \mathcal{D}_{p}\right), f \in \mathcal{M}$, and $g \in \mathcal{M}^{\perp} \subset \mathcal{D}_{q}$. Then for all $\lambda \in \mathbb{D}$,

$$
\int_{|z|=1} \frac{\lambda}{z-\lambda}\left(D_{z}(f, g)+f(z) \bar{g}(z)\right) \frac{|d z|}{2 \pi}=0 .
$$

Proof. Let $\lambda \in \mathbb{D}$. Then

$$
\begin{aligned}
& \lambda \int_{|z|=1} D_{z}\left(\frac{f-f(\lambda)}{z-\lambda}, g\right) \frac{|d z|}{2 \pi} \\
= & \lambda \int_{|z|=1} \int_{|\zeta|=1} \frac{1}{z-\zeta}\left(\frac{f(z)-f(\lambda)}{z-\lambda}-\frac{f(\zeta)-f(\lambda)}{\zeta-\lambda}\right)\left(\frac{\bar{g}(z)-\bar{g}(\zeta)}{\bar{z}-\bar{\zeta}}\right) \frac{|d \zeta|}{2 \pi} \frac{|d z|}{2 \pi} \\
= & \lambda \int_{|z|=1} \int_{|\zeta|=1} \frac{(\zeta-\lambda) f(z)-(\zeta-\lambda) f(\lambda)-(z-\lambda) f(\zeta)+(z-\lambda) f(\lambda)}{(z-\zeta)(z-\lambda)(\zeta-\lambda)} \\
= & \int_{|z|=1} \frac{\lambda f(\lambda)}{z-\lambda} \int_{|\zeta|=1} \frac{1}{\zeta-\lambda}\left(\frac{\bar{g}(z)-\bar{g}(\zeta)}{\bar{z}-\bar{\zeta}}\right) \frac{|d \zeta|}{2 \pi} \frac{|d z|}{2 \pi} \\
& +\int_{|z|=1} \frac{\lambda}{z-\lambda}\left(\int_{|\zeta|=1}\left(\frac{f(z)-f(\zeta)}{z-\zeta}\right) \frac{|d \zeta|}{2 \pi} \frac{|d z|}{2 \pi}\right. \\
& +\int_{|\zeta|=1} \frac{f(\zeta)}{\lambda-\zeta}\left(\frac{\bar{g}(z)-\bar{g}(\zeta)}{\bar{z}-\bar{\zeta}) \frac{|d \zeta|}{2 \pi}}\right.
\end{aligned}
$$


Notice that

$$
\begin{aligned}
\int_{|\zeta|=1} & \frac{1}{\zeta-\lambda}\left(\frac{\bar{g}(z)-\bar{g}(\zeta)}{\bar{z}-\bar{\zeta}}\right) \frac{|d \zeta|}{2 \pi} \\
= & \int_{|\zeta|=1} \frac{1}{1-\lambda \bar{\zeta}} \bar{\zeta}\left(\frac{\bar{g}(z)-\bar{g}(\zeta)}{\bar{z}-\bar{\zeta}}\right) \frac{|d \zeta|}{2 \pi} \\
= & 0 \quad[|d \zeta|] \text {-a.e. } z
\end{aligned}
$$

since the integrand above belongs to $\bar{\zeta} H^{q}$. Also notice that

$$
\begin{aligned}
\int_{|z|=1} & \frac{\lambda}{z-\lambda} \int_{|\zeta|=1} \frac{f(\zeta)}{\lambda-\zeta}\left(\frac{\bar{g}(z)-\bar{g}(\zeta)}{\bar{z}-\bar{\zeta}}\right) \frac{|d \zeta|}{2 \pi} \frac{|d z|}{2 \pi} \\
= & \int_{|\zeta|=1} \frac{\lambda f(\zeta)}{\lambda-\zeta} \int_{|z|=1} \frac{1}{z-\lambda}\left(\frac{\bar{g}(z)-\bar{g}(\zeta)}{\bar{z}-\bar{\zeta}}\right) \frac{|d z|}{2 \pi} \frac{|d \zeta|}{2 \pi} \\
= & \int_{|\zeta|=1} \frac{\lambda f(\zeta)}{\lambda-\zeta} \int_{|z|=1} \frac{\bar{z}}{1-\lambda \bar{z}}\left(\frac{\bar{g}(z)-\bar{g}(\zeta)}{\bar{z}-\bar{\zeta}}\right) \frac{|d z|}{2 \pi} \frac{|d \zeta|}{2 \pi} \\
= & 0
\end{aligned}
$$

since the integrand in the inner integral above belongs to $\bar{z} \overline{H^{q}}$. Thus

$$
\lambda \int_{|z|=1} D_{z}\left(\frac{f-f(\lambda)}{z-\lambda}, g\right) \frac{|d z|}{2 \pi}=\int_{|z|=1} \frac{\lambda}{z-\lambda} D_{z}(f, g) \frac{|d z|}{2 \pi} .
$$

By (2.5) and (7.1)

$$
0=\lambda \int_{|z|=1} D_{z}\left(\frac{f-f(\lambda)}{z-\lambda}, g\right) \frac{|d z|}{2 \pi}+\lambda \int_{|z|=1} \frac{f-f(\lambda)}{z-\lambda} \bar{g}(z) \frac{|d z|}{2 \pi} .
$$

By (7.2), this reduces to

$$
0=\int_{|z|=1} \frac{\lambda}{z-\lambda}\left(D_{z}(f, g)+f \bar{g}\right) \frac{|d z|}{2 \pi}-\lambda f(\lambda) \int_{|z|=1} \frac{\bar{g}(z)}{z-\lambda} \frac{|d z|}{2 \pi} .
$$

But notice that

$$
\int_{|z|=1} \frac{\bar{g}(z)}{z-\lambda} \frac{|d z|}{2 \pi}=\int_{|z|=1} \frac{\bar{z}}{1-\lambda \bar{z}} \bar{g}(z) \frac{|d z|}{2 \pi}=0
$$


since the above integrand belongs to $\bar{z} \overline{H^{q}}$. Thus we have

$$
0=\int_{|z|=1} \frac{\lambda}{z-\lambda}\left(D_{z}(f, g)+f \bar{g}\right) \frac{|d z|}{2 \pi}
$$

which completes the proof.

Lemma 7.4. Let $\mathcal{M} \in \operatorname{Lat}\left(L, \mathcal{D}_{p}\right)$, and let $f \in \mathcal{M}, g \in \mathcal{M}^{\perp} \subset \mathcal{D}_{q}, g \neq 0$. Then for $[|d \zeta|]$-a.e. $w \in \mathbb{T}$ we have

$$
\left\langle\frac{\lambda}{z-\lambda}, g\right\rangle\left(c_{\lambda}(f, g)-f(w)\right) \rightarrow 0 \quad \text { as } \lambda \rightarrow w \text { non-tangentially, }|\lambda|>1 .
$$

Proof. Let $J$ be the set of points $w \in \mathbb{T}$ such that $D_{p, w}(f), D_{q, w}(g)$, and hence $D_{w}(f, g)$ are finite. Note that $J$ has full measure in $\mathbb{T}$ and from our previous discussion, the non-tangential limits of $f$ and $g$ exist for all $w \in J$.

We have to show that for $[|d \zeta|]$-a.e. $w \in J$

$$
\left\langle\frac{z f}{z-\lambda}, g\right\rangle-\left\langle\frac{\lambda}{z-\lambda}, g\right\rangle f(w) \rightarrow 0, \quad \text { as } \lambda \rightarrow w \text { non-tangentially, }|\lambda|>1 .
$$

We substitute $1 / \bar{\lambda}$ for $\lambda$, set

$$
H(\lambda)=\left\langle\frac{f}{1-\bar{\lambda} z}, g\right\rangle,
$$

and note that for $\lambda \in \mathbb{D}$

$$
\left\langle\frac{1}{1-\bar{\lambda} z}, g\right\rangle=\overline{(z g)^{\prime}(\lambda)}
$$

Moreover

$$
\left\langle\frac{\bar{\lambda} z f}{1-\bar{\lambda} z}, g\right\rangle=\left\langle\frac{f}{1-\bar{\lambda} z}, g\right\rangle=H(\lambda)
$$

since $\langle f, g\rangle=0$. Thus, we must show that for $[|d \zeta|]$-a.e. $w \in J$,

$$
H(\lambda)-\overline{(z g)^{\prime}(\lambda)} f(w) \rightarrow 0 \quad \text { as } \lambda \rightarrow w \text { non-tangentially. }
$$

To prove this, we notice from Lemma 7.3 that

$$
H(\lambda)=\left\langle\frac{f}{1-\bar{\lambda} z}, g\right\rangle+\int_{|z|=1} \frac{\lambda}{z-\lambda}\left(D_{z}(f, g)+f \bar{g}\right) \frac{|d z|}{2 \pi} .
$$

The Poisson kernel $P_{\lambda}(z)(\lambda \in \mathbb{D}, z \in \mathbb{T})$ is equal to

$$
P_{\lambda}(z)=\frac{\lambda \bar{z}}{1-\lambda \bar{z}}+\frac{1}{1-\bar{\lambda} z} .
$$


Thus, using Lemma 2.1 of [38] and (7.3), we get that for $w \in J$

$$
\begin{aligned}
H(\lambda)=\int_{|z|=1} & P_{\lambda}(z)\left(D_{z}(f, g)+f \bar{g}\right) \frac{|d z|}{2 \pi} \\
& +\int_{|z|=1} \frac{\bar{\lambda} z}{(1-\bar{\lambda} z)^{2}}(f(z)-f(w))(\bar{g}(z)-\bar{g}(w)) \frac{|d z|}{2 \pi}+f(w) \overline{\lambda g^{\prime}(\lambda)} .
\end{aligned}
$$

By Fatou's theorem, the first term in the above equation goes to $D_{w}(f, g)+$ $f(w) \bar{g}(w)$ for almost all $w \in J$ as $\lambda$ approaches $w$ non-tangentially. For the second term we note that

$$
\begin{aligned}
\int_{|z|=1} & \frac{\bar{\lambda} z}{(1-\bar{\lambda} z)^{2}}(f(z)-f(w))(\bar{g}(z)-\bar{g}(w)) \frac{|d z|}{2 \pi} \\
= & \int_{|z|=1} \frac{\bar{\lambda} z}{(1-\bar{\lambda} z)^{2}}|z-w|^{2} \frac{(f(z)-f(w))(\bar{g}(z)-\bar{g}(w))}{|z-w|^{2}} \frac{|d z|}{2 \pi} .
\end{aligned}
$$

Because

$$
\frac{\bar{\lambda} z}{(1-\bar{\lambda} z)^{2}}|z-w|^{2} \rightarrow-1
$$

as $\lambda \rightarrow w$ non-tangentially, we can use the dominated convergence theorem to get that the above converges to $-D_{w}(f, g)$.

Putting all this together, we get that

$$
H(\lambda)-\overline{(z g)^{\prime}(\lambda)} f(w) \rightarrow 0
$$

almost everywhere on $\mathbb{T}$ as $\lambda \rightarrow w$ non-tangentially.

Proof of Theorem 7.1. Let $f, g \neq 0$, and $E \subset \mathbb{T}$ be as in the statement of the theorem. If $F(\lambda)=c_{\lambda}(f, g)$ converges non-tangentially to $F(w)$ on $E$, and if $F(w) \neq f(w)$ on some subset $A \subset E$ with $|A|>0$, then by Lemma 7.4, the analytic function

$$
\left\langle\frac{\lambda}{z-\lambda}, g\right\rangle, \quad \lambda \in \mathbb{D}_{e}
$$

would have non-tangential boundary values which are equal to zero $[|d \zeta|]$-a.e. on $A$. By Privalov's theorem this would imply that $\left\langle(z-\lambda)^{-1}, g\right\rangle=0$ for each $\lambda \in \mathbb{D}_{e}$, but this would mean that $g \equiv 0$, which contradicts the choice of $g$.

For a function $g \in \mathcal{D}_{q}$ we use $[g]_{L^{*}}$ to denote the smallest $L^{*}$-invariant subspace of $\mathcal{D}_{q}$. 
Corollary 7.5. Let $g \in \mathcal{D}_{q}$ such that $(z g)^{\prime}$ has finite non-tangential limits on some set $E \subset \mathbb{T}$ with $|E|>0$. Then every $f \in \mathcal{M}={ }^{\perp}[g]_{L^{*}}$ has a pseudocontinuation $\tilde{f}$ across E. Furthermore, this pseudocontinuation is given by

$$
\tilde{f}(\lambda)=c_{\lambda}(f, g)=\lambda\left\langle\frac{f}{\lambda-z}, g\right\rangle / \overline{(z g)^{\prime}(1 / \bar{\lambda})} .
$$

In particular, for each $\lambda \in \mathbb{D}_{e}$ with $(z g)^{\prime}(1 / \bar{\lambda}) \neq 0, f \rightarrow \tilde{f}(\lambda)$ defines a bounded linear functional on $\mathcal{M}$.

Proof. As in the proof of Lemma 7.4, one verifies that for $\lambda \in \mathbb{D}_{e}$,

$$
\left\langle\frac{\lambda}{z-\lambda}, g\right\rangle=-\overline{(z g)^{\prime}(1 / \bar{\lambda})}
$$

Thus, the hypothesis implies that $\left\langle(z-\lambda)^{-1}, g\right\rangle$ remains bounded as $\lambda$ approaches $E$ non-tangentially, and so by Lemma $7.4 c_{\lambda}(f, g)$ approaches $f(w)$ non-tangentially $[|d \zeta|]$-a.e. on $E$, i.e., $c_{\lambda}(f, g)$ is a pseudocontinuation of $f$ across E.

Notice that an easy calculation shows that if $g \in \mathcal{D}_{q}$ then for every $f \in$ ${ }^{\perp}[g]_{L^{*}}$ and every polynomial $p$, one has

$$
c_{\lambda}(f, g)=c_{\lambda}\left(f, p\left(L^{*}\right) g\right)
$$

and hence $c_{\lambda}(f, h)$ is independent of $h \in[g]_{L^{*}}$, and so $\sigma\left(\left.L\right|^{\perp}[g]_{L^{*}}\right) \cap \mathbb{D}$ is discrete for any non-zero $g \in \mathcal{D}_{q}$. We have not been able to show under the hypothesis of the previous Corollary that $\sigma(L \mid \mathcal{N}) \cap \mathbb{D}$ is discrete for any non-zero $\mathcal{N} \in$ $\operatorname{Lat}\left(L, \mathcal{D}_{p}\right)$ with $\mathcal{N} \subset{ }^{\perp}[g]_{L^{*}}$.

Corollary 7.6. Let $g, h \in \mathcal{D}_{q}$ and set $\mathcal{M}={ }^{\perp}[g]_{L^{*}} \cap{ }^{\perp}[h]_{L^{*}}$. If $(z g)^{\prime}(\lambda) /(z h)^{\prime}(\lambda)$ has a finite non-tangential limit on a set $E \subset \mathbb{T},|E|>0$, then $\sigma(L \mid \mathcal{M}) \cap \mathbb{D}$ is discrete.

Proof. We note that the hypothesis implies that $h \neq 0$, and that the conclusion is trivial if $g=0$. Thus, we assume that $g, h \neq 0$. If we let

$$
P=\left\{\lambda \in \mathbb{D}_{e}:(z g)^{\prime}(1 / \bar{\lambda})=0 \quad \text { or }(z h)^{\prime}(1 / \bar{\lambda})=0\right\},
$$

then

$$
c_{\lambda}(f, g)-c_{\lambda}(f, h)=\frac{\langle z f /(\lambda-z), g\rangle}{\overline{(z g)^{\prime}(1 / \bar{\lambda})}}-\frac{\langle z f /(\lambda-z), h\rangle}{\overline{(z h)^{\prime}(1 / \bar{\lambda})}}
$$

is meromorphic in $\mathbb{D}_{e}$ with possible poles at the points $\lambda \in P$. We shall first show that for any $f \in \mathcal{M}, c_{\lambda}(f, g)=c_{\lambda}(f, h)$ for every $\lambda \in \mathbb{D}_{e} \backslash P$. 
We have for $[|d \zeta|]$-a.e. $w \in E$

$$
\begin{aligned}
& \left\langle\frac{\lambda}{z-\lambda}, g\right\rangle\left(c_{\lambda}(f, g)-c_{\lambda}(f, h)\right) \\
= & \left\langle\frac{\lambda}{z-\lambda}, g\right\rangle\left(c_{\lambda}(f, g)-f(w)\right)-\frac{\overline{(z g)^{\prime}(1 / \bar{\lambda})}}{\overline{(z h)^{\prime}(1 / \bar{\lambda})}}\left\langle\frac{\lambda}{z-\lambda}, h\right\rangle\left(f(w)-c_{\lambda}(f, h)\right) .
\end{aligned}
$$

Thus, by Lemma 7.4, our hypothesis, and Privalov's theorem, the meromorphic function

$$
\left\langle\frac{\lambda}{z-\lambda}, g\right\rangle\left(c_{\lambda}(f, g)-c_{\lambda}(f, h)\right)
$$

equals zero in $\mathbb{D}_{e}$, and this implies $c_{\lambda}(f, g)=c_{\lambda}(f, h)$ for all $\lambda \in \mathbb{D}_{e} \backslash P$. We note that $\mathcal{M}^{\perp}=[g]_{L^{*}} \vee[h]_{L^{*}}$. Thus, by Proposition 2.8 and the above, in order to show that $\sigma(L \mid \mathcal{M}) \cap \mathbb{D}$ is discrete it suffices to show that

$$
c_{\lambda}\left(f, p\left(L^{*}\right) g+q\left(L^{*}\right) h\right)=c_{\lambda}(f, g)=c_{\lambda}(f, h)
$$

for all $\lambda \in \mathbb{D}_{e} \backslash P$ and all polynomials $p$ and $q$. This is a straightforward calculation.

The $H^{2}$-duality, Proposition 5.9 (with $\mathfrak{B}=\mathcal{D}_{p}$ and $\mathfrak{D}=L_{a}^{q}$ ), and the previous Corollary immediately imply the following result.

Corollary 7.7. Let $1<q<+\infty$, and let $G, H \in L_{a}^{q}$ such that $G / H$ has a finite non-tangential limit on a set $E \subset \mathbb{T},|E|>0$, then $\operatorname{ind}[G, H]=1$.

\section{REFERENCES}

1. E. Авакumov, Cyclicity and approximation by lacunary power series, Michigan Math. J. 42 (1995), 277-299.

2. A. AleKsandrov, Invariant subspaces of the backward shift operator in the Smirnov class, Linear and Complex Analysis Problem Book, Lecture Notes in Mathematics, Number 1043, V.P. Havin, S.V. Hruscev, and N.K. Nikol'skii, eds., Springer-Verlag, New York, 1984.

3. A. AleKsandrov, Invariant subspaces of the backward shift operator in the space $H^{p}$ $(p \in(0,1))$, Investigations on linear operators and the theory of functions, IX, Zap. Naucn. Sem. Leningrad Otdel. Math. Inst. Steklov (LOMI), 92 (1979), 7-29.

4. A. Aleksandrov, Invariant subspaces of shift operators. An axiomatic approach, Investigations on linear operators and the theory of functions, XI, Zap. Naucn. Sem. Leningrad Otdel. Math. Inst. Steklov (LOMI), 113 (1981), 7-26.

5. A. Aleman, Hilbert spaces of analytic functions between the Hardy space and the Dirichlet space, Proc. Amer. Math. Soc. 115 (1992), 97-104.

6. A. Aleman And S. Richter, Some sufficient conditions for the division property of invariant subspaces in weighted Bergman spaces, J. Funct. Anal. 144 (1997), 542-556.

7. A. Aleman, S. Richter, And C. Sundberg, Beurling's theorem for the Bergman space, Acta Math. 177 (1996), 275-310. 
8. A. Aleman And W. T. Ross, The backward shift on weighted Bergman spaces, Mich. Math. J., 43 (1996), 291-319.

9. S. Axler, Zero multipliers of Bergman spaces, Canad. Math. Bull. 28 (1985), 237-242.

10. S. AxLER, Bergman spaces and their operators. Surveys of some recent results in operator theory, Vol. I, 1-50, Pitman Res. Notes Math. Ser., 171, Longman Sci. Tech., Harlow, 1988.

11. H. Bercovici, C. Foiaş, And C. Pearcy, Dual algebras with applications to invariant subspaces and dilation theory, CBMS Regional Conf. Ser. in Math., no. 56, Amer. Math. Soc., Providence, RI., 1985.

12. A. Beurling, On two problems concerning linear transformations in Hilbert space, Acta Math. 81 (1949), 239-255.

13. P. Bourdon, Cellular-indecomposable shifts and Beurling's theorem, Michigan Math. J. 33 (1986), 187-193.

14. L. Brown, A. Shields, AND K. Zeller, On absolutely convergent exponential sums, Trans. Amer. Math. Soc. 96 (1960), 162-183.

15. L. Carleson, On the zeros of functions with bounded Dirichlet integrals, Math. Z. 56 (1952), 289-295.

16. J.B. Conway, The Theory of Subnormal Operators, Mathematical Surveys and Monographs, Vol 36, American Mathematical Society, Providence R.I., 1991.

17. J.B. Conway, Functions of One Complex Variable II, Springer-Verlag, New York, 1995.

18. A.E. Durbashian And F.A. Shamoian, Topics in the theory of $A_{\alpha}^{p}$ spaces, B.G. Tuebner, Verlagsgesellschasft, Leipzig, 1988.

19. J. Douglas, Solution of the problem of Plateau, Trans. Amer. Math. Soc. 33 (1931), 263-321.

20. R.G. Douglas, H.S. Shapiro, And A.L. Shields, Cyclic vectors and invariant subspaces for the backward shift operator, Ann. Inst. Fourier (Grenoble), 20 (1970), 37-76.

21. P. L. Duren, Theory of $H^{p}$ spaces, Pure and Appl. Math., Vol. 38, Academic Press, New York, 1970.

22. J. EschmeIER, Multiplication operators on Bergman spaces are reflexive, Op. Thry. Adv. Appl. 43 (1990), 165-184.

23. S. D. Fisher, Function Theory on Planar Domains, Wiley, New York, 1983.

24. J. B. Garnett, Bounded Analytic Functions, Pure and Appl. Math., Vol. 96, Academic Press, New York, 1981.

25. J. B. Garnett, Analytic Capacity and Measure, Lecture Notes in Mathematics, \# 297, Springer-Verlag, New York, 1972.

26. H. Hedenmalm, An invariant subspace of the Bergman space having the co-dimension two property, J. Reine Angew. Math. 443 (1993), 1-9.

27. H. Hedenmalm, S. Richter, K. Seip, Interpolating sequences and invariant subspaces of given index in the Bergman spaces, J. Reine Angew. Math. 477 (1996), 13-30.

28. C. Horowitz, Zeros of functions in the Bergman spaces, Duke Math. J. 41 (1974), 693-710.

29. J.P.KAhANe AND Y. KATZnelson, Sur le comportement radial des fonctions analytiques, Comptes rend. Acad. Scie. Paris, Serie A, t. 272, 15 mars 1971, 718-719.

30. M. V. Keldys, Sur l'approximation en moyenne par polynomes de functions d'une variable complexe, Mat. Sb. 16 (58), 1945, 1-20.

31. S. Khrushchev And V. Peller, Hankel operators, best approximation, and stationary Gaussian processes, Russian Math. Surveys, 37 (1982), 61-144.

32. P. Koosis, Introduction to $H_{p}$ Spaces, London Math. Soc. Lecture Notes, 40, Cambridge Univ. Press, Cambridge, 1980.

33. N. Nikol'SkiI, Treatise on the Shift Operator, Springer-Verlag, New York, 1986. 
34. R. F. Olin AND L. YANG, The commutant of multiplication by $z$ on the closure of polynomials in $L^{t}(\mu)$, J. Funct. Anal. 134 (1995), 297-320.

35. S. RichteR, Invariant subspaces in Banach spaces of analytic functions, Trans. Amer. Math. Soc. 304 (1987), 585-616.

36. S. Richter, Invariant subspaces of the Dirichlet shift, J. Reine Angew. Math. 386 (1988), 205-220.

37. S. Richter And A. Shields, Bounded analytic functions in the Dirichlet space, Math. Z. 198 (1988), 151-159.

38. S. Richter And C. Sundberg, Invariant subspaces of the Dirichlet shift and pseudocontinuations, Trans. Amer. Math. Soc. 341 (1994), 863-879.

39. S. Richter And C. Sundberg, A formula for the local Dirichlet integral, Michigan Math. J. 38 (1991), 355-379.

40. W. Rudin, Function theory in the unit ball of $\mathbb{C}^{n}$, Springer-Verlag, New York, 1980

41. K. SEIP, Beurling-type density theorems in the unit disk, Invent. Math. 113 (1993), 26-39.

42. H.S. Shapiro, Generalized analytic continuation. Symposia on Theor. Phys. and Math. 8 (1968), Plenum Press, New York, 151-163.

43. E.M. Stein, Singular Integrals and Differentiability Properties of Functions, Princeton Press, Princeton, New Jersey, 1970.

44. B.A. Taylor and D.L. Williams, Zeros of Lipschitz functions in the unit disc, Mich. Math. J. 18 (1971), 129-139.

45. J. E. Thomson, Approximation in the mean by polynomials, Ann. Math. 133 (1991), 477-507.

46. J. E. ThOmSOn And L. YAnG, Invariant subspaces with the codimension one property in $L^{t}(\mu)$, Indiana Univ. Math. J. 44 (1995), 1163-1173.

47. S. WALSH, Cyclic vectors for the backward Bergman shift, Ph.D. dissertation, University of California, Berkeley, 1984.

48. L. YANG, Invariant subspaces of the Bergman space and some subnormal operators in $\mathbb{A}_{1} \backslash \mathbb{A}_{2}$, Mich. Math. J, 42 (1995), 301-310.

49. L. YANG, Boundary values of functions in $P^{t}$ spaces, Houston J. Math., 21 (1995), $465-472$.

Research by the last two authors was supported, in part, by the National Science Foundation, and was done, in part, while visiting Fernuniversitaet Hagen.

Alexandru Aleman

Fachbereich Mathematik

Fernuniversität Hagen

Postfach 940

58084 Hagen, GERMANY

Stefan Richter

Department of Mathematics

University of Tennessee

Knoxville, Tennessee 37996-1300

E-MAIL: richter@novell.math.utk.edu
A. Aleman's Current address:

Department of Mathematics and Statistics

Case Western Reserve University

Cleveland, Ohio 43403, U. S. A.

E-MAIL: Alexandru.Aleman@FernUni-Hagen.de

William T. Ross

Department of Mathematics and Computer Science

University of Richmond

Richmond, Virginia 23173

E-MAIL: wross@richmond.edu

Received: February 12th, 1998; revised: May 13th, 1998. 